\title{
Complexity growth of rotating black holes with a probe string
}

\author{
Koichi Nagasaki ${ }^{*}$ \\ School of Physics, University of Electronic Science and Technology of China (UESTC), \\ No. 4, Section II, North Jianshe Road, Chengdu 610054, China
}

(Received 12 July 2018; published 27 December 2018)

\begin{abstract}
We study the effect of a probe string to black hole complexity according to the complexity equals action conjecture. Our system contains a particle moving on the boundary of black hole spacetime. In the dual description this corresponds to the insertion of a fundamental string on the bulk spacetime. The total action consists of the Einstein-Hilbert term and the Nambu-Goto term. The effect of this string is expressed by the Nambu-Goto term. Focusing on the Nambu-Goto term, we analyze the time development of this system. Our results show some interesting properties of complexity. This gives us a useful hint for defining complexity in quantum field theories.
\end{abstract}

DOI: 10.1103/PhysRevD.98.126014

\section{INTRODUCTION}

The concept of computational complexity was originally known in quantum information theory [1-7] or computer science $[8,9]$. This concept gave us a new physical quantity to study gravitational physics. An important goal of quantum gravity is to reveal the inside of the black hole horizon or the information problem with black holes [10-19]. A candidate for its solution is firewalls [20-22]. Complexity is realized as an important quantity for studying the structure of black hole spacetime - the Einstein-Rosen bridge [23-31], which is a structure of connecting two external black holes thought to be equivalent to an entangled pair of particles $(E R=E P R)$ [32]. Specifically, complexity is thought to be a good tool of diagnosing the existence of firewalls [31]. Because of these motivations, complexity has been studied in many recent works [25,33-43].

The definite approach for quantifying complexity is still unknown. So to define complexity in quantum field theory is a theme of recent research [44-49]. In the perspective of quantum information theory, complexity is roughly defined as the number of necessary gates which operate to produce the target state from the reference (initial) state. The tensor network is frequently used in a quantum system [50-55], and it is used for describing the wormhole structure [56]. Then it seems to be a good approach for defining complexity. There is also a geometric approach for defining complexity [57]. A geometric approach is

\footnotetext{
*koichi.nagasaki24@gmail.com
}

Published by the American Physical Society under the terms of the Creative Commons Attribution 4.0 International license. Further distribution of this work must maintain attribution to the author(s) and the published article's title, journal citation, and DOI. Funded by SCOAP. suggested to introduce Finsler geometry on quantum space [58-63]. In this approach complexity is determined by the geodesic on the quantum space.

The holographic duality [64] has been an important principle in recent research. According to this principle, complexity is expected to have a holographic dual in the gravity theory. That relation between a gravity theory and a quantum field theory has been a recent active research theme [65-71]. The complexity-action (CA) conjecture $[72,73]$ is the most reliable candidate for this duality. This conjecture suggests a relation between computational complexity and the gravitational action which is evaluated in a specific region of spacetime called a Wheeler-DeWitt (WDW) patch. CA conjecture has been tested in various spacetime settings [74-95]. Complexity growth of some kinds of black holes, especially Kerr-AdS ones, are calculated in Ref. [96]. In general there is a divergence of action. Treating the boundary terms of the gravitational action and its renormalization is one of the important problems in proving this conjecture. For this purpose the Neumann boundary term for gravity [97] and other solutions have been considered so far [78,98-105]. That conjecture in the time dependent system [74,106-111] is our main interest here. In Ref. [109] especially, the counterpart in a field theory is discussed for Finsler geometry and the Fubini-Study metric.

Some of the property of complexity is found in recent works. For example, it has a good analogy with entropy in thermodynamics: it satisfies the second law of thermodynamics [112]. The time development of complexity satisfies the Lloyd bound $[73,113,114]$. Specifically, in Ref. [114] complexity in the process of the formation of the black holes is discussed. Furthermore, interestingly, Ref. [115] revealed that complexity has a nonlocal property. 
The analysis of complexity using a probe is a useful methodFor example, in Ref. [116] complexity growth in a system with flavor branes is studied, and a nonlocal operator in a Bañados-Teitelboim-Zanelli (BTZ) black hole is studied in Ref. [117]. And also complexity of a particle falling in the Poincare-AdS boundary is studied in the probe approximation in Ref. [118]. In this paper we use this kind of method. Our probe is a fundamental string. The Einstein-Hilbert action of various kinds of spacetime is calculated in many works. On the other hand we study the effect of the probe string here. The total action of this system is the sum of the Einstein-Hilbert action and the Nambu-Goto (NG) action of the string. The NG action is also obtained by integrating over the WDW patch. The motivation for studying such an effect is found in Refs. [119-125], where energy loss of the charged quarks is calculated by considering a drag force caused by the string motion.

In a previous paper [126] we studied the effect of the probe string moving on the $\mathrm{AdS}_{3+1}$ black hole spacetime. What I have found about complexity so far is as follows:

(i) Complexity basically grows as the black hole mass becomes larger.

(ii) But in the vicinity of light speed complexity shows a specific behavior.

(iii) Complexity is smaller as the probe string moves faster.

The most notable result is the last one. I think it can be stated that a fast moving object decreases the growth of the complexity. This result may serve as a hint for finding the definition of complexity in quantum field theory. In this paper we try to find new properties of complexity by studying the effects of the probe string on a more broad type of black hole.

This paper is organized as follows. In Sec. II we begin with calculating the effect of the probe string on the AdS black hole. This is a higher-dimensional generalization of the previous work. We first compute the general $n$-dimensional case and reproduce the $(4+1)$-dimensional results. And then $(3+1)$ - and $(5+1)$-dimensional results are also found. In Sec. III we study the NG action of a string moving in three-dimensional black hole spacetime. In this section a new parameter-angular momentum - is introduced. This black hole is the BTZ black hole [127]. The angular momentum will show an interesting phenomena which is not found in our previous work. In Sec. IV the angular momentum is added to the AdS black holes. This is the KerrAdS black hole. Their complexity growth is studied in Ref. [96]. The drag force of the four-dimensional Kerr-AdS black hole is studied in Ref. [128]. In their case, the drag force is located in the boundary of the AdS. In our case, on the other hand, we take into account the inner part of the black hole horizon. We review this analysis and also study the five-dimensional case. In the final section, Sec. V, we summarize our results and remark on new insights about them. After that some future directions are suggested.

\section{AdS $_{n+1}$ BLACK HOLES}

In this section we study the cases of an AdS black hole in an arbitrary dimension. The $n=3$ case will reproduce a previous work [126]. Here we consider noncharged black holes. This metric is

$$
\begin{aligned}
d s_{\mathrm{AdS}_{n+1}}^{2} & =-f(r) d t^{2}+\frac{d r^{2}}{f(r)}+r^{2} d \Omega_{n-1}, \\
f(r) & =1-\frac{8 \pi}{(n-1) \Omega_{n-1}} \frac{2 G M}{r^{n-2}}+\frac{r^{2}}{\ell_{\mathrm{AdS}}^{2}} \\
& =1-\frac{r_{\mathrm{m}}^{n-2}}{r^{n-2}}+\frac{r^{2}}{\ell_{\mathrm{AdS}}^{2}}, \\
r_{\mathrm{m}}^{n-2} & :=\frac{16 \pi G M}{(n-1) \Omega_{n-1}} .
\end{aligned}
$$

The volume of the ( $n-1)$-sphere is $\Omega_{n-1}=2 \pi^{n / 2} / \Gamma(n / 2)$. In each dimension the relation between $r_{\mathrm{m}}$ and mass is from $r_{\mathrm{m}}^{n-2}=16 \pi G M /\left((n-1) \Omega_{n-1}\right)$. For later use, we write them here explicitly in four, five, and six dimensions:

$$
\begin{gathered}
(3+1)-\operatorname{dim}: r_{\mathrm{m}}=\frac{8 \pi M}{\Omega_{2}}=2 M \\
(4+1)-\operatorname{dim}: r_{\mathrm{m}}=\left(\frac{16 \pi M}{3 \Omega_{3}}\right)^{1 / 2}=\left(\frac{8 M}{3 \pi}\right)^{1 / 2}, \\
(5+1)-\operatorname{dim}: r_{\mathrm{m}}=\left(\frac{4 \pi M}{\Omega_{4}}\right)^{1 / 3}=\left(\frac{3 M}{2 \pi}\right)^{1 / 3}
\end{gathered}
$$

As before we assume that the string moves a great circle on $S^{n-1}$ subspace. Then the induced metric of this part is the same as before $d \Omega_{n-1}=d \phi^{2}$. We take the world sheet parameter as (21)

$$
t=\tau, \quad r=\sigma, \quad \phi=v \tau+\xi(\sigma) .
$$

As before we scale $r$ so that $\ell_{\text {AdS }}=1$. In the following $t$, $r_{\mathrm{m}}, M$, and the world sheet coordinate $\sigma$ are rescaled in the same way. Then $\ell_{\text {AdS }}$ disappears in the expression, and the metric is rescaled to the original one times $\ell_{\text {AdS }}^{2}$. The induced metric is

$$
\begin{aligned}
d s_{\mathrm{AdS}_{n+1}}^{2} \text { ind }= & \left(-f(\sigma)+\sigma^{2} v^{2}\right) d \tau^{2} \\
& +\left(\frac{1}{f(\sigma)}+\sigma^{2} \xi^{\prime}(\sigma)^{2}\right) d \sigma^{2}+2 \sigma^{2} v \xi^{\prime}(\sigma) d \tau d \sigma \\
f(\sigma)=1 & -\left(\frac{r_{\mathrm{m}}}{\sigma}\right)^{n-2}+\sigma^{2} .
\end{aligned}
$$

The NG action is obtained by integrating over the WDW patch, 


$$
\begin{aligned}
\frac{d S_{\mathrm{NG}}}{d t} & =T_{\mathrm{s}} \int_{0}^{r_{\mathrm{h}}} d \sigma \sqrt{-g_{\mathrm{ind}}(\sigma)} \\
& =T_{\mathrm{s}} \int_{0}^{r_{\mathrm{h}}} d \sigma \sqrt{1-\frac{v^{2} \sigma^{2}}{f(\sigma)}+\sigma^{2} f(\sigma) \xi^{\prime}(\sigma)^{2}} \\
& =: \int_{0}^{r_{\mathrm{h}}} d \sigma \mathcal{L}_{\operatorname{AdS}(n+1)},
\end{aligned}
$$

where the horizon $r_{\mathrm{h}}$ is determined by $f(r)=0$. Here we comment on the horizon. By differentiating $f(r)$,

$$
f^{\prime}(r)=(n-2) \frac{r_{\mathrm{m}}}{r^{n-1}}+2 r ; \quad n \geq 3,
$$

we find that $f(r)$ is a monotonically increasing function. This fact and that this function takes a negative value near $r=0$ mean that the equation $f(r)=0$ certainly has only one positive solution.

EOM and its solution-The equation of motion for $\xi$ gives

$$
0=\frac{d}{d \sigma}\left(\frac{\sigma^{2} f(\sigma) \xi^{\prime}(\sigma)}{\sqrt{1-v^{2} \sigma^{2} / f(\sigma)+\sigma^{2} f(\sigma) \xi^{\prime}(\sigma)^{2}}}\right) .
$$

From this equation the constant $c_{\xi}$ is defined as follows,

$$
c_{\xi}:=\frac{\sigma^{2} f(\sigma) \xi^{\prime}(\sigma)}{\mathcal{L}_{\mathrm{AdS}(n+1)} / T_{\mathrm{S}}},
$$

by solving it for $\xi^{\prime}(\sigma)$,

$$
\xi^{\prime}(\sigma)=\frac{c_{\xi}}{\sigma^{2} f(\sigma)} \sqrt{\frac{\sigma^{2} f(\sigma)-v^{2} \sigma^{4}}{\sigma^{2} f(\sigma)-c_{\xi}^{2}}} .
$$

The constant $c_{\xi}$ is determined in the same way as before from the real valued condition. The zero of the numerator gives the equation

$f(\sigma)-v^{2} \sigma^{2}=0 \Rightarrow\left(1-v^{2}\right) \sigma^{n}+\sigma^{n-2}-r_{\mathrm{m}}^{n-2}=0$.

The function on the left-hand side is a monotonically increasing function of $\sigma$ (assuming that $n \geq 3$ ) and takes a negative value at $\sigma=0$. Then this function has only one solution for $\sigma>0$. We call it $\sigma_{\mathrm{H}}:\left(1-v^{2}\right) \sigma_{\mathrm{H}}^{n}+\sigma_{\mathrm{H}}^{n-2}-$ $r_{\mathrm{m}}^{n-2}=0$. Since the denominator becomes zero at the same value of $\sigma, \sigma=\sigma_{\mathrm{H}}$, the constant $c_{\xi}$ is determined:

$0=\sigma_{\mathrm{H}}^{2} f\left(\sigma_{\mathrm{H}}\right)-c_{\xi}^{2}=v^{2} \sigma_{\mathrm{H}}^{4}-c_{\xi}^{2}, \quad \therefore c_{\xi}=v \sigma_{\mathrm{H}}^{2}$.
In the above the second equality is derived from numerator condition (10). We assumed that $c_{\xi}$ is positive. We obtain

$$
\xi^{\prime}(\sigma)=\frac{c_{\xi}}{\sigma^{2} f(\sigma)} \sqrt{\frac{\sigma^{n-2} f(\sigma)-v^{2} \sigma^{n}}{\sigma^{n-2} f(\sigma)-v^{2} \sigma_{\mathrm{H}}^{4} \sigma^{n-4}}} .
$$

Action-The NG action is obtained by integrating over the WDW patch:

$$
\frac{1}{T_{\mathrm{S}}} \int_{0}^{r_{\mathrm{h}}} d \sigma \mathcal{L}_{\mathrm{AdS}(n+1)}=\int_{0}^{r_{\mathrm{h}}} d \sigma \sqrt{\frac{\sigma^{n-2} f(\sigma)-v^{2} \sigma^{n}}{\sigma^{n-2} f(\sigma)-v^{2} \sigma_{\mathrm{H}}^{4} \sigma^{n-4}}} .
$$

This form is a general form for $n \geq 3$. In the following we focus concretely on four, five, and six dimensions.

\section{A. AdS $_{3+1}$ case}

In $(3+1)$ dimensions Eq. (13) is

$$
\begin{aligned}
\xi^{\prime}(\sigma) & =\frac{c_{\xi}}{\sigma^{2} f(\sigma)} \sqrt{\frac{\sigma f(\sigma)-v^{2} \sigma^{3}}{\sigma f(\sigma)-v^{2} \sigma_{\mathrm{H}}^{4} / \sigma}} \\
f(\sigma) & =1-\frac{r_{\mathrm{m}}}{\sigma}+\sigma^{2}
\end{aligned}
$$

By construction the numerator and the denominator have the common factor. Then the expression can be simplified:

$$
\begin{aligned}
& \sigma f(\sigma)-v^{2} \sigma^{3}-\left(\sigma_{\mathrm{H}} f\left(\sigma_{\mathrm{H}}\right)-v^{2} \sigma_{\mathrm{H}}^{3}\right) \\
& \quad=\left(\sigma-\sigma_{\mathrm{H}}\right)\left(1+\left(1-v^{2}\right)\left(\sigma^{2}+\sigma_{\mathrm{H}} \sigma+\sigma_{\mathrm{H}}^{2}\right)\right), \\
& \sigma f(\sigma)-v^{2} \sigma_{\mathrm{H}}^{4} / \sigma-\left(\sigma_{\mathrm{H}} f\left(\sigma_{\mathrm{H}}\right)-v^{2} \sigma_{\mathrm{H}}^{3}\right) \\
& \quad=\left(\sigma-\sigma_{\mathrm{H}}\right)\left(1+\left(\sigma^{2}+\sigma_{\mathrm{H}} \sigma+\sigma_{\mathrm{H}}^{2}\right)+v^{2} \sigma_{\mathrm{H}}^{4} /\left(\sigma \sigma_{\mathrm{H}}\right)\right) .
\end{aligned}
$$

Then the development of the NG action is

$$
\begin{aligned}
& \frac{1}{T_{\mathrm{s}}} \frac{d S_{\mathrm{NG}}}{d t} \\
& \quad=\int_{0}^{r_{\mathrm{h}}} d \sigma \sqrt{\frac{1+\left(1-v^{2}\right)\left(\sigma^{2}+\sigma \sigma_{\mathrm{H}}+\sigma_{\mathrm{H}}^{2}\right)}{1+\left(\sigma^{2}+\sigma \sigma_{\mathrm{H}}+\sigma_{\mathrm{H}}^{2}\right)+v^{2} \sigma_{\mathrm{H}}^{3} / \sigma}} \\
& \quad=\int_{0}^{r_{\mathrm{h}}} d \sigma \sqrt{\frac{\left(1-v^{2}\right)\left(\sigma^{3}+\sigma_{\mathrm{H}} \sigma^{2}\right)+\left(\left(1-v^{2}\right) \sigma_{\mathrm{H}}^{2}+1\right) \sigma}{\sigma^{3}+\sigma_{\mathrm{H}} \sigma^{2}+\left(\sigma_{\mathrm{H}}^{2}+1\right) \sigma+v^{2} \sigma_{\mathrm{H}}^{3}}} .
\end{aligned}
$$

By numerical calculation this action can be expressed as a function of $M$ and $v$. Recall that the black hole mass is given by Eqs. (14) and (3). The result is shown in Figs. 1 and 2. 


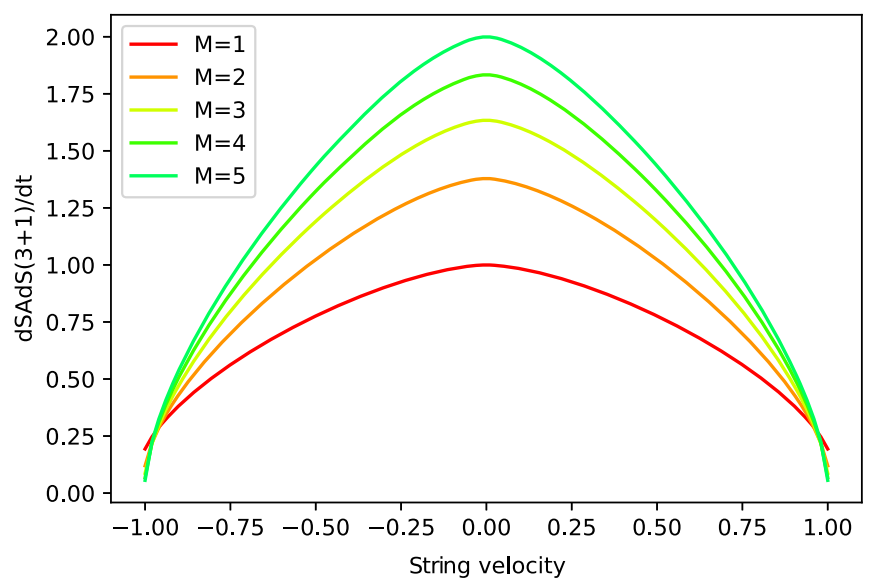

FIG. 1. $\operatorname{AdS}_{3+1}$ : Action growth vs string velocity.

Figure 1 shows the velocity dependence. As usual this dependence takes a maximum when the string is stationary.

Figure 2 shows the mass dependence. There are notable behaviors here. One is a peak at lower mass. The other is a phase transition. For slower strings its effect increases according to mass increasing. But for fast strings, especially near light speed, it changes to a decreasing function of the mass.

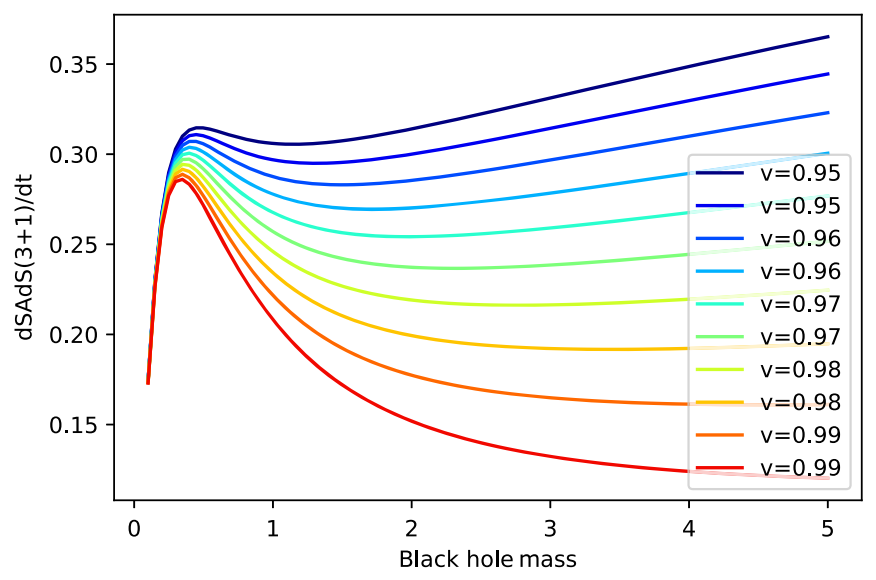

FIG. 2. $\operatorname{AdS}_{3+1}$ : Action growth vs black hole mass.

\section{B. AdS $_{4+1}$ case}

When $n=4$, Eq. (13) becomes

$$
\frac{1}{T_{\mathrm{s}}} \frac{d S_{\mathrm{NG}}}{d t}=\int_{0}^{r_{\mathrm{h}}} d \sigma \sqrt{\frac{\left(1-v^{2}\right)\left(\sigma^{2}+\sigma_{\mathrm{H}}^{2}\right)+1}{\sigma^{2}+\sigma_{\mathrm{H}}^{2}+1}} .
$$

As studied in Ref. [126], this integral is performed with an elliptic integral. We show here the result again:

$$
\begin{aligned}
\frac{d S_{\mathrm{NG}}}{d t}= & -i T_{\mathrm{s}}\left(\frac{1+\sqrt{1+4 r_{\mathrm{m}}^{2}\left(1-v^{2}\right)}}{2}\right)^{1 / 2} \\
& \times E\left[\arcsin \left(i\left(\frac{\left(-1+\sqrt{4 r_{\mathrm{m}}^{2}+1}\right)\left(1-v^{2}\right)}{\left(1-2 v^{2}\right)+\sqrt{1+4 r_{\mathrm{m}}^{2}\left(1-v^{2}\right)}}\right)^{1 / 2}\right),\left(\frac{\left(1-2 v^{2}\right)+\sqrt{1+4 r_{\mathrm{m}}^{2}\left(1-v^{2}\right)}}{1+\sqrt{1+4 r_{\mathrm{m}}^{2}\left(1-v^{2}\right)}}\right)^{1 / 2}\right],
\end{aligned}
$$

where $r_{m}$ is related to the black hole mass by $4 r_{\mathrm{m}}^{2}=32 M /(3 \pi)$, as noted in Eq. (3). The velocity dependence and the mass dependence are shown in Figs. 3 and 4. It reproduces the results in the previous work [126].

\section{AdS $_{5+1}$ case}

In the $(5+1)$-dimensional case Eq. (13) is, explicitly,

$$
\frac{1}{T_{\mathrm{s}}} \frac{d S_{\mathrm{NG}}}{d t}=\int_{0}^{r_{\mathrm{h}}} d \sigma \sqrt{\frac{\left(\sigma^{2}+\sigma_{\mathrm{H}} \sigma+\sigma_{\mathrm{H}}^{2}\right)+\left(1-v^{2}\right)\left(\sigma^{4}+\sigma_{\mathrm{H}} \sigma^{3}+\sigma_{\mathrm{H}}^{2} \sigma^{2}+\sigma_{\mathrm{H}}^{3} \sigma+\sigma_{\mathrm{H}}^{4}\right)}{\left(\sigma^{2}+\sigma_{\mathrm{H}} \sigma+\sigma_{\mathrm{H}}^{2}\right)+\left(\sigma^{4}+\sigma_{\mathrm{H}} \sigma^{3}+\sigma_{\mathrm{H}}^{2} \sigma^{2}+\sigma_{\mathrm{H}}^{3} \sigma+\sigma_{\mathrm{H}}^{4}\right)-v^{2} \sigma_{\mathrm{H}}^{4}}} .
$$

This integral is also performed by the numerical calculation method. The velocity dependence and the mass dependence are shown in Figs. 5 and 6. Remarkable points of these plots are as follows.

For Fig. 5, the curve of the velocity dependence is gentler compared to the four- and five-dimensional cases (Figs. 1 and 3). Compared with the lower-dimensional cases, the effect of the probe string decreases slower at higher velocity. Specifically, it does not reach zero at the light velocity, while it becomes zero in the BTZ black hole case (see Figs. 7 and 8). We can say that the effect on complexity becomes insensitive as the dimensionality is higher. It can also be seen from the fact that the maximum value is lower than the $\mathrm{AdS}_{3+1}$ and $\mathrm{AdS}_{4+1}$ cases.

For the mass dependence shown in Fig. 6, the maximum at the vicinity of light speed disappears here. This is now a monotonically increasing function of mass in all regions of mass and velocity. 


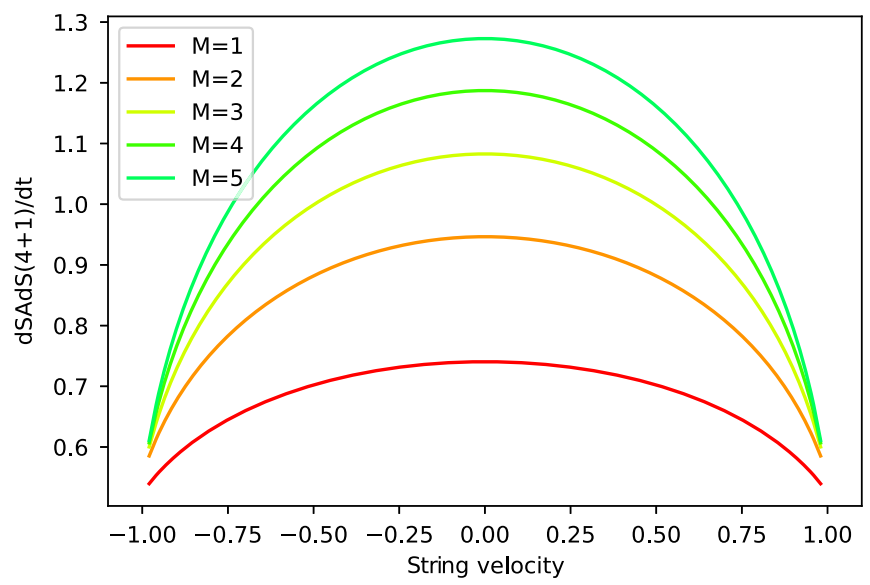

FIG. 3. $\mathrm{AdS}_{4+1}$ : Action growth vs string velocity.

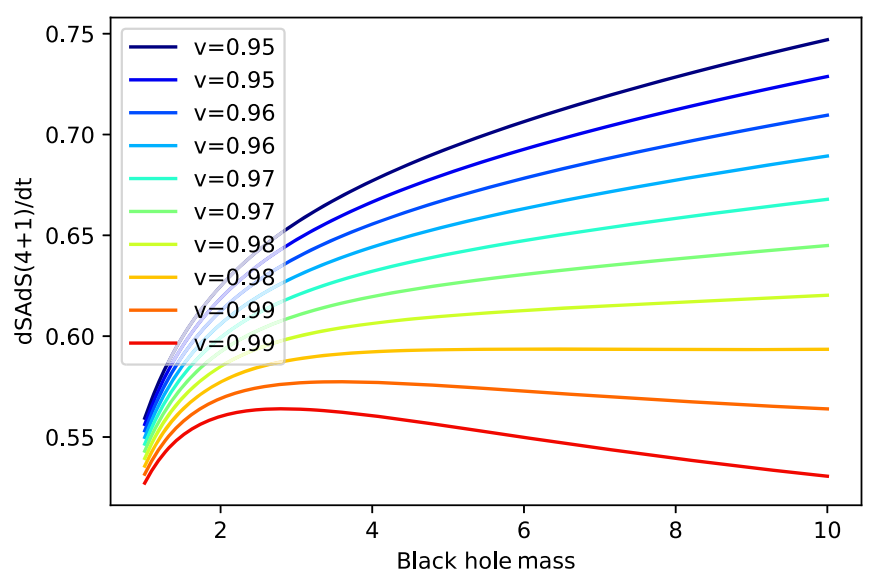

FIG. 4. $\mathrm{AdS}_{4+1}$ : Action growth vs black hole mass.

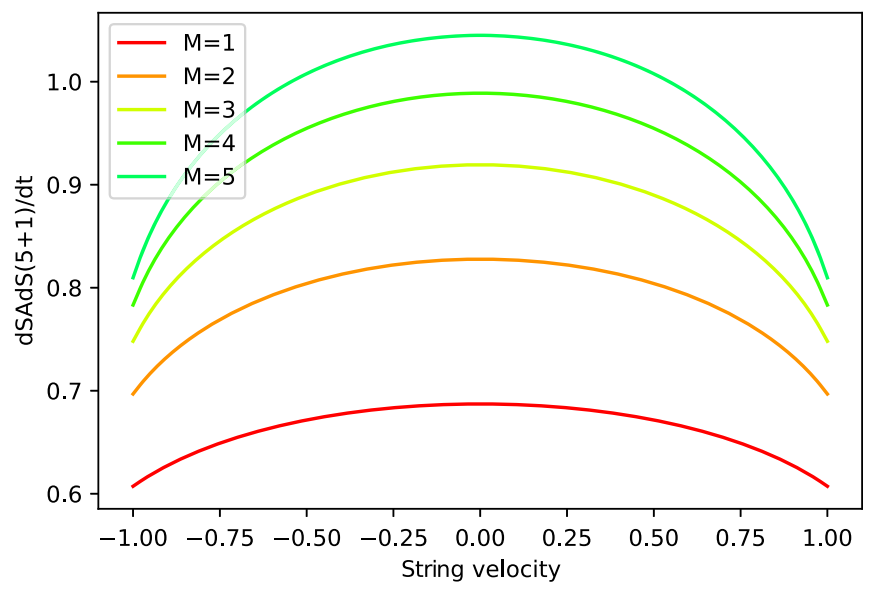

FIG. 5. $\mathrm{AdS}_{5+1}$ : Action growth vs string velocity.

We expect that this behavior is a general tendency in higher than six dimensions. That is, the dependence between different masses and velocities becomes smaller in higher dimensions.

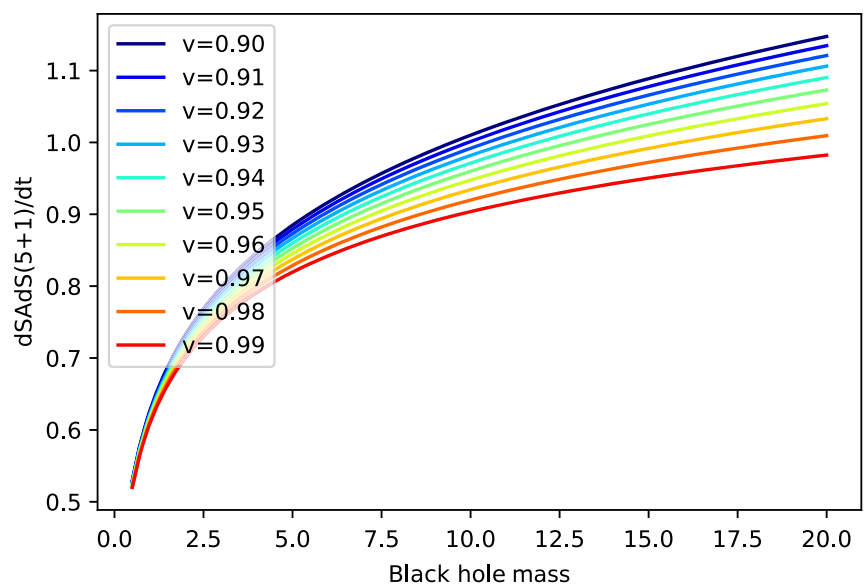

FIG. 6. $\mathrm{AdS}_{5+1}$ : Action growth vs black hole mass.

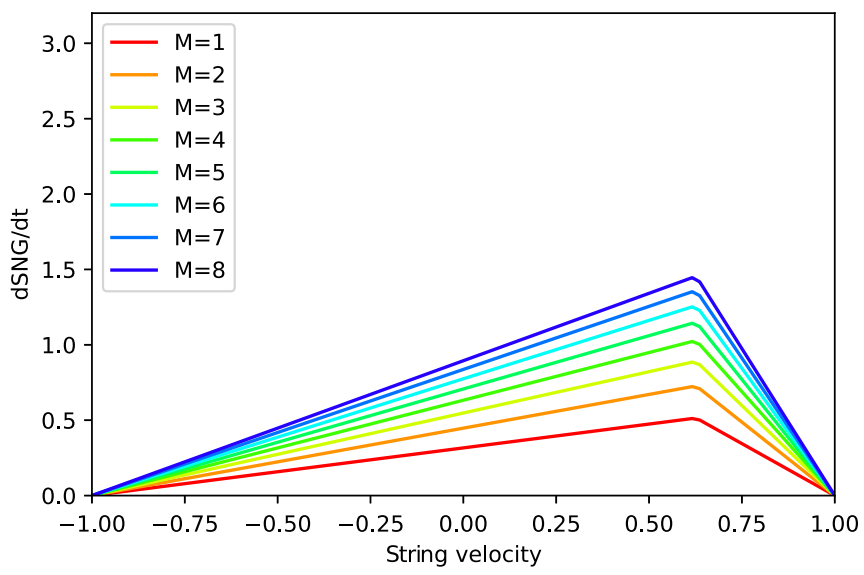

FIG. 7. BTZ: Action growth vs fixed string velocity $J / M=0.9$.

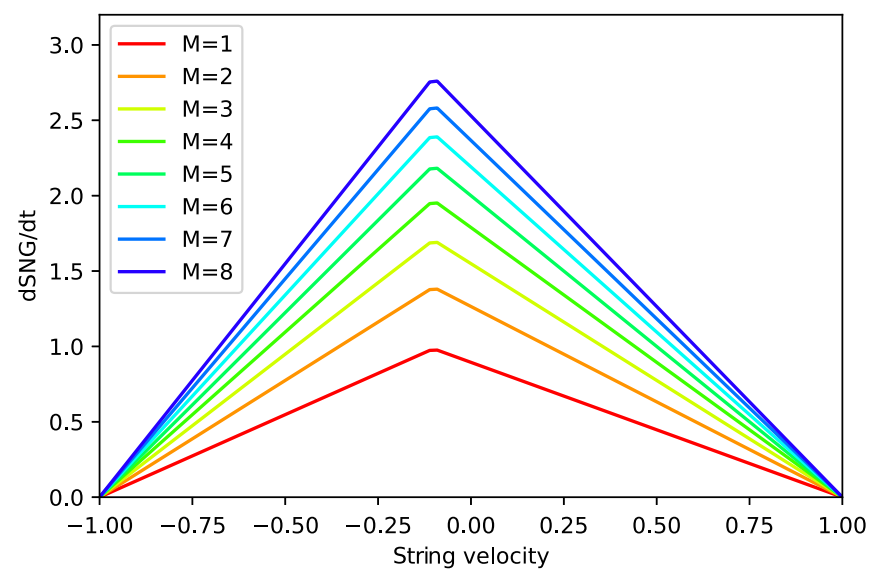

FIG. 8. BTZ: Action growth vs fixed string velocity $J / M=-0.2$.

\section{BTZ BLACK HOLES}

We consider the string moving in the BTZ black hole spacetime. The butterfly effects caused by a small 
perturbation on an asymptotic region in this black hole is studied in Ref. [129]. In this section we study the effect of the string moving on this spacetime geometry. The BTZ black hole is $(2+1)$-spacetime specified as

$$
\begin{aligned}
d s_{\mathrm{BTZ}}^{2} & =-f(r) d t^{2}+\frac{d r^{2}}{f(r)}+r^{2}\left(d \phi-\frac{r_{+} r_{-}}{\ell_{\mathrm{AdS}} r^{2}} d t\right)^{2}, \\
f(r) & :=\frac{\left(r^{2}-r_{+}^{2}\right)\left(r^{2}-r_{-}^{2}\right)}{\ell_{\mathrm{AdS}}^{2} r^{2}} .
\end{aligned}
$$

The parameters $r_{ \pm}$are the inner and the outer horizon, which are related to black hole mass $M$ and angular momentum $J$ by

$$
M=\left(r_{+}^{2}+r_{-}^{2}\right) / \ell_{\mathrm{AdS}}^{2}, \quad J=2 r_{+} r_{-} / \ell_{\mathrm{AdS}}
$$

We rescale $r_{\text {(old) }}=r_{\text {(new) }} \ell_{\text {AdS }}$ and for $r_{ \pm}$and $t$ in the same way. This simplifies the expression and the metric becomes the original one times $\ell_{\text {AdS }}^{2}$. One edge of the string moves with velocity $v$. We parametrize the world sheet as follows:

$$
t=\tau, \quad r=\sigma, \quad \phi=v \tau+\xi(\sigma) .
$$

Since we use here the $\ell_{\text {AdS }}=1$ unit, the angular velocity of the string $\omega$ is $\omega=v / \ell_{\mathrm{AdS}}=v$. The induced metric is

$$
\begin{aligned}
& d s_{\mathrm{BTZind}}^{2}=-\left(f(\sigma)-\left(v \sigma-\frac{r_{+} r_{-}}{\sigma}\right)^{2}\right) d \tau^{2} \\
&+\left(\frac{1}{f(\sigma)}+\sigma^{2} \xi^{\prime}(\sigma)^{2}\right) d \sigma^{2} \\
&+2\left(v \sigma-\frac{r_{+} r_{-}}{\sigma}\right) \sigma \xi^{\prime}(\sigma) d \tau d \sigma \\
& f(\sigma)=\frac{\left(\sigma^{2}-r_{+}^{2}\right)\left(\sigma^{2}-r_{-}^{2}\right)}{\sigma^{2}}
\end{aligned}
$$

The Nambu-Goto Lagrangian is given by the determinant of this metric,

$$
\mathcal{L}_{\mathrm{BTZ}}=T_{\mathrm{s}} \sqrt{1+f(\sigma) \sigma^{2} \xi^{\prime}(\sigma)^{2}-\frac{1}{f(\sigma)}\left(v \sigma-\frac{J}{2 \sigma}\right)^{2}},
$$

where angular momentum is rescaled as $J_{(\text {old })} / \ell_{\text {AdS }}=: J$.

EOM and its solution-By the equation of motion,

$$
\frac{d}{d \sigma}\left(\frac{f(\sigma) \sigma^{2} \xi^{\prime}(\sigma)}{\mathcal{L}_{\mathrm{BTZ}} / T_{\mathrm{S}}}\right)=0, \quad c_{\xi}:=\frac{f(\sigma) \sigma^{2} \xi^{\prime}(\sigma)}{\mathcal{L}_{\mathrm{BTZ}} / T_{\mathrm{s}}},
$$

$$
\xi^{\prime}(\sigma)=\frac{c_{\xi}}{\sigma^{2} f(\sigma)} \sqrt{\frac{\sigma^{2} f(\sigma)-\left(v \sigma^{2}-J / 2\right)^{2}}{\sigma^{2} f(\sigma)-c_{\xi}^{2}}} .
$$

For this function to give the real values, the numerator and the denominator in the square root must have the same zero point. This condition leads that the denominator is zero when

$$
\sigma=\sigma_{\mathrm{H}}^{2}:=\frac{M-v J}{1-V^{2}}
$$

This determines the integration constant as

$$
c_{\xi}=\left|v \sigma_{\mathrm{H}}^{2}-J / 2\right| .
$$

Then the square root of Eq. (26) is factorized by $\left(\sigma-\sigma_{\mathrm{H}}\right)$ :

$\xi^{\prime}(\sigma)=\frac{c_{\xi}}{\sigma^{2} f(\sigma)} \sqrt{\frac{\left(1-v^{2}\right)\left(\sigma^{2}+\sigma_{\mathrm{H}}^{2}\right)-(M-v J)}{\sigma^{2}+\sigma_{\mathrm{H}}^{2}-M}}$.

From the relation (25) the Lagrangian is

$$
\frac{\mathcal{L}_{\mathrm{BTZ}}}{T_{\mathrm{S}}}=\sqrt{\frac{\left(1-v^{2}\right) \sigma^{2}}{\sigma^{2}+\sigma_{\mathrm{H}}^{2}-M}}
$$

Action-The development of the Nambu-Goto action is obtained by integrating this Lagrangian over the WDW patch,

$$
\begin{aligned}
\frac{1}{T_{\mathrm{s}}} \frac{d S_{\mathrm{NG}}}{d t} & =\sqrt{1-v^{2}} \int_{r_{-}}^{r_{+}} d \sigma \frac{\sigma}{\sqrt{\sigma^{2}+\sigma_{\mathrm{H}}^{2}-M}} \\
& =\left|r_{+}-v r_{-}\right|-\left|r_{-}-v r_{+}\right| .
\end{aligned}
$$

In our scaling, $\quad M=r_{+}^{2}+r_{-}^{2}, J=2 r_{+} r_{-}, \quad r_{ \pm}=$ $\frac{1}{2}(\sqrt{M+J} \pm \sqrt{M-J})$. We express the above action by the parameter $M$ and $J$ :

$$
\begin{aligned}
\frac{1}{T_{\mathrm{s}}} \frac{d S_{\mathrm{NG}}}{d t}= & \frac{1}{2}((1-v) \sqrt{M+J}+(1+v) \sqrt{M-J}) \\
& -\frac{1}{2}|(1-v) \sqrt{M+J}-(1+v) \sqrt{M-J}| .
\end{aligned}
$$

This plot is shown in Figs. 7, 8, 9, and 10.

According to the results in the previous work [126], complexity growth is expected to take the maximum when the string is stationary. So it seems to be meaningful to see the dependence of the relative velocity between the black 


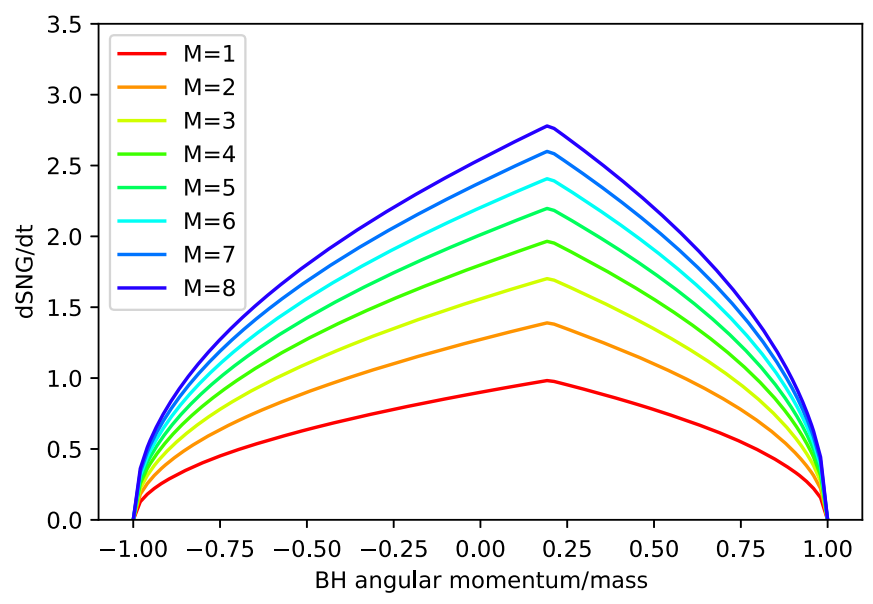

FIG. 9. BTZ: Action growth vs black hole angular momentum/ mass.

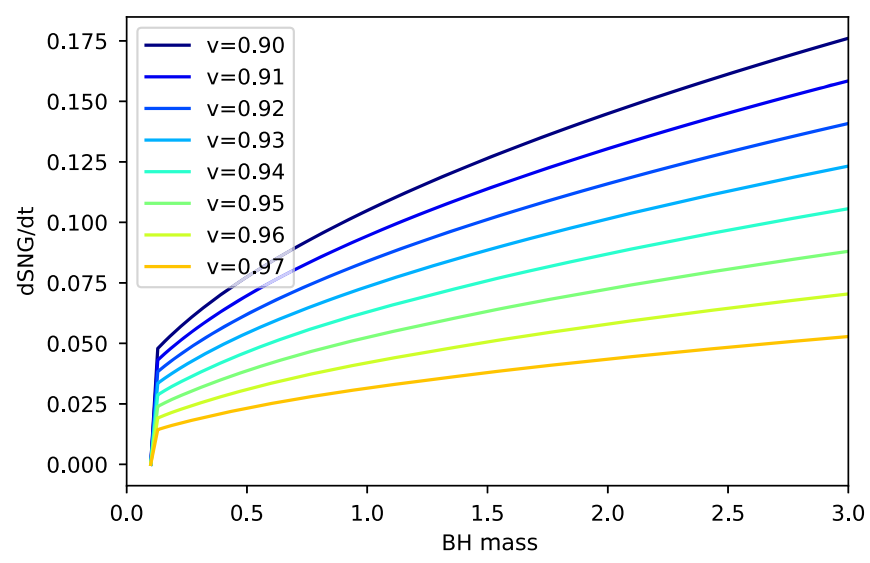

FIG. 10. BTZ: Action growth vs black hole mass (small mass region).

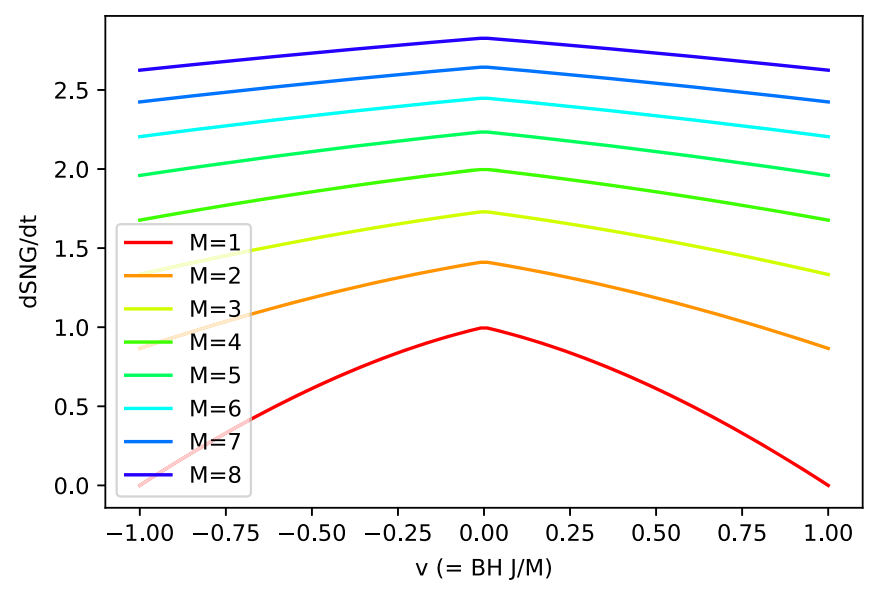

FIG. 11. BTZ: Action growth vs string velocity (relative velocity $=0)$.

hole and the string. That dependence of the relative velocity is shown in Figs. 11 and 12. As expected, this tendency is seen in these plots. That is, the effect on complexity takes

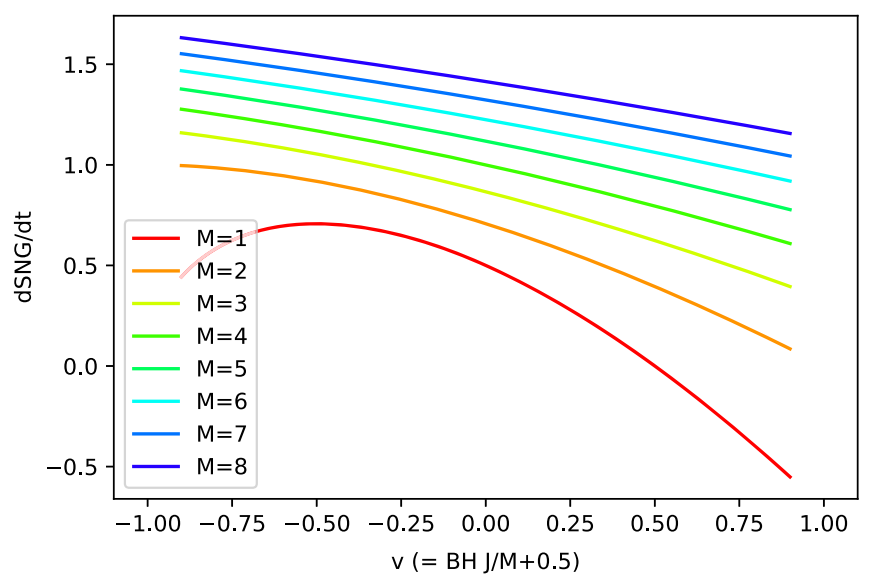

FIG. 12. BTZ: Action growth vs string velocity (relative velocity $=0.5)$.

the maximum when the relative velocity is zero for rotating black holes. The effect is larger for larger black holes in the $\mathrm{AdS}_{4}$ case [126].

Figures 7 and 8 show the velocity dependence for different angular momentums $J / M$. In Fig. 7 the peak position is shifted because of the black hole rotation. In Fig. 8, since the black hole rotates in the opposite direction to the string, the peak is shifted to the opposite side. Not only is the peak position shifted, we can also see that the peak value becomes smaller for a large shift. Note that in this case the effect of the probe string is exactly zero when the string motion reaches light speed.

Figure 10 also shows that the effect of the string is smaller for fast moving strings. And this is a monotonically increasing function of the black hole mass. It can be thought that this is because complexity defines how complex the physical system is. Then a larger system may have more information.

\section{KERR-AdS BLACK HOLES}

In this section we consider the Kerr-AdS black holes which are black hole solutions with angular momentum. A general higher-dimensional solution is known, and that holographic correspondence is studied in Refs. [130,131].

\section{A. Four-dimensional Kerr-AdS black holes}

We consider here AdS black holes with angular momentum. The Einstein-Hilbert action of these black holes is studied in Ref. [96]. We study an effect of the probe string here. In the Boyer-Lindquist coordinates the fourdimensional Kerr-AdS black hole is given by

$$
\begin{aligned}
d s_{\mathrm{KA} 4}^{2}= & -\frac{\Delta_{r}}{\rho^{2}}\left(d t-\frac{a \sin ^{2} \theta}{\Xi} d \phi\right)^{2}+\frac{\rho^{2}}{\Delta_{r}} d r^{2}+\frac{\rho^{2}}{\Delta_{\theta}} d \theta^{2} \\
& +\frac{\Delta_{\theta} \sin ^{2} \theta}{\rho^{2}}\left(a d t-\frac{r^{2}+a^{2}}{\Xi} d \phi\right)^{2}
\end{aligned}
$$




$$
\begin{aligned}
= & -\left(\frac{\Delta_{r}}{\rho^{2}}-\frac{\Delta_{\theta} \sin ^{2} \theta}{\rho^{2}} a^{2}\right) d t^{2}+\frac{\rho^{2}}{\Delta_{r}} d r^{2}+\frac{\rho^{2}}{\Delta_{\theta}} d \theta^{2} \\
& +2 \frac{a \Delta_{r} \sin ^{2} \theta-a\left(r^{2}+a^{2}\right) \Delta_{\theta} \sin ^{2} \theta}{\rho^{2} \Xi} d t d \phi \\
& +\frac{\left(r^{2}+a^{2}\right)^{2} \Delta_{\theta} \sin ^{2} \theta-a^{2} \Delta_{r} \sin ^{4} \theta}{\rho^{2} \Xi^{2}} d \phi^{2},
\end{aligned}
$$

where

$$
\begin{aligned}
& \Delta_{r}=\left(r^{2}+a^{2}\right)\left(1+r^{2} / \ell_{\mathrm{AdS}}^{2}\right)-2 m r, \quad \Xi=1-a^{2} / \ell_{\mathrm{AdS}}^{2}, \\
& \Delta_{\theta}=1-a^{2} \cos ^{2} \theta / \ell_{\mathrm{AdS}}^{2}, \quad \rho^{2}=r^{2}+a^{2} \cos ^{2} \theta .
\end{aligned}
$$

The physical mass and the angular momentum are $M=$ $m /\left(G_{\mathrm{N}} \Xi\right)$ and $J=m a /\left(G_{\mathrm{N}} \Xi\right)$. The above metric is related to the AdS coordinates $\Phi$ [see also Eq. (4.11) of Ref. [132] ] as follows. Put the AdS boundary coordinates as $t$ and $\Omega$, and they are related by $\Phi=\phi+\Omega t$. In this coordinate the first and last terms of Eq. (33) are, at $\theta=\pi / 2$,

$$
\begin{aligned}
-r^{2} \ell_{\mathrm{AdS}}^{-2}\left[d t-\frac{a}{\Xi} d \phi\right]^{2} & =-r^{2} \ell_{\mathrm{AdS}}^{-2}\left[\left(1-\frac{a \Omega}{\Xi}\right) d t-\frac{a}{\Xi} d \Phi\right]^{2}, \\
\frac{1}{r^{2}}\left[a d t-\frac{r^{2}}{\Xi} d \phi\right]^{2} & =\frac{1}{r^{2}}\left[\left(a-\frac{r^{2} \Omega}{\Xi}\right) d t-\frac{r^{2}}{\Xi} d \Phi\right]^{2} .
\end{aligned}
$$

In order for these terms to give the form of the AdS metric at $r \rightarrow \infty$, the cross terms from these terms should cancel. Then the parameter $\Omega$ is determined:

$$
\phi=\Phi-a \ell_{\mathrm{AdS}}^{-2} t .
$$

We consider a string moving in this spacetime. In the following we use the rescaled coordinates such that $\ell_{\text {AdS }}=1$. We assume that the string moves on the great circle of the subspace $S^{2}$ : that is, $\theta=\pi / 2$. We parametrize the string world sheet as ${ }^{1}$

$$
t=\tau, \quad r=\sigma, \quad \Phi=V \tau+\xi(\sigma) .
$$

Taking into account the relation (35), the above is

$$
t=\tau, \quad r=\sigma, \quad \phi=(V-a) \tau+\xi(\sigma):=v \tau+\xi(\sigma) .
$$

We defined the shifted velocity as $v:=V-a$, which is used in the following calculation, while the original $V$ is the string velocity. Let us define a function

$$
\Delta(\sigma):=\Delta_{r}(r=\sigma)=\left(\sigma^{2}+a^{2}\right)\left(1+\sigma^{2}\right)-2 m \sigma .
$$

\footnotetext{
${ }^{1}$ In this section we use the capital letters $\Phi$ and $V$ for the boundary coordinate and the string velocity. These are shifted because of the rotation of the black hole.
}

The induced metric is

$$
\begin{aligned}
d s_{\text {KA4ind }}^{2}= & -\left(\frac{\Delta}{\sigma^{2}}-\frac{a^{2}}{\sigma^{2}}-2 \frac{a v}{\sigma^{2}} \frac{\Delta-\left(\sigma^{2}+a^{2}\right)}{\Xi}\right. \\
& \left.-v^{2} \frac{\left(\sigma^{2}+a^{2}\right)^{2}-a^{2} \Delta}{\sigma^{2} \Xi^{2}}\right) d \tau^{2} \\
& +\left(\frac{\sigma^{2}}{\Delta}+\frac{\left(\sigma^{2}+a^{2}\right)^{2}-a^{2} \Delta}{\sigma^{2} \Xi^{2}} \xi^{\prime 2}\right) d \sigma^{2} \\
& +\frac{2 \xi^{\prime}}{\sigma^{2} \Xi}\left(a\left(\Delta-\left(\sigma^{2}+a^{2}\right)\right)\right. \\
& \left.+v \frac{\left(\sigma^{2}+a^{2}\right)^{2}-a^{2} \Delta}{\Xi}\right) d \tau d \sigma .
\end{aligned}
$$

We define the functions for simplicity:

$F:=\Delta-\left(\sigma^{2}+a^{2}\right), \quad G:=\left(\sigma^{2}+a^{2}\right)^{2}-a^{2} \Delta$.

Its determinant is

$$
\begin{aligned}
-\operatorname{det}\left[g_{\mathrm{KA} 4 \text { ind }}\right]= & \left(\frac{\Delta}{\sigma^{2}}-\frac{a^{2}}{\sigma^{2}}-2 \frac{a v}{\sigma^{2}} \frac{F}{\Xi}-v^{2} \frac{G}{\sigma^{2} \Xi^{2}}\right) \\
& \times\left(\frac{\sigma^{2}}{\Delta}+\frac{G}{\sigma^{2} \Xi^{2}} \xi^{\prime 2}\right)+\frac{\xi^{\prime 2}}{\sigma^{4} \Xi^{2}}\left(a F+v \frac{G}{\Xi}\right)^{2} .
\end{aligned}
$$

Further we define

$$
\begin{aligned}
H(\sigma) & :=\frac{\Delta}{\sigma^{2}}-\frac{a^{2}}{\sigma^{2}}-2 \frac{a v}{\sigma^{2}} \frac{F}{\Xi}-v^{2} \frac{G}{\sigma^{2} \Xi^{2}}, \\
I(\sigma) & :=\left(a F+v \frac{G}{\Xi}\right)^{2} .
\end{aligned}
$$

EOM and Lagrangian-The Lagrangian and the equation of motion is

$$
\begin{aligned}
\frac{\mathcal{L}_{\mathrm{KA} 4}}{T_{\mathrm{s}}} & =\left[H\left(\frac{\sigma^{2}}{\Delta}+\frac{G}{\sigma^{2} \Xi^{2}} \xi^{\prime 2}\right)+\frac{\xi^{\prime 2}}{\sigma^{4} \Xi^{2}} I\right]^{1 / 2}, \\
\frac{1}{T_{\mathrm{s}}} \frac{\partial \mathcal{L}_{\mathrm{KA} 4}}{\partial \xi^{\prime}(\sigma)} & =\frac{\xi^{\prime} /\left(\sigma^{4} \Xi^{2}\right)}{\mathcal{L}_{\mathrm{KA} 4} / T_{\mathrm{s}}}\left[\sigma^{2} H G+I\right]=: c_{\xi} .
\end{aligned}
$$

Solving it for $\xi^{\prime}(\sigma)$, we obtain

$\xi^{\prime}=c_{\xi} \sigma^{4} \Xi^{2} \sqrt{\frac{\sigma^{2} H / \Delta}{\left(\sigma^{2} H G+I\right)\left(\sigma^{2} H G+I-c_{\xi}^{2} \sigma^{4} \Xi^{2}\right)}}$.

We impose the reality condition as before. We need to find the zero of 


$$
\begin{aligned}
\sigma^{2} H & =\Delta-a^{2}-2 a v \frac{\Delta-\left(\sigma^{2}+a^{2}\right)}{\Xi}-v^{2} \frac{\left(\sigma^{2}+a^{2}\right)^{2}-a^{2} \Delta}{\Xi^{2}} \\
& =\Delta(\sigma)\left(1-\frac{a v}{\Xi}\right)^{2}-\left(a-\frac{v\left(\sigma^{2}+a^{2}\right)}{\Xi}\right)^{2}
\end{aligned}
$$

We denote this solution as $\sigma=\sigma_{\mathrm{H}}$. Actually $\sigma^{2} H(\sigma)=0$ has a unique solution in the positive region. The denominator must be zero, coincidentally. This condition determines the integration constant $c_{\xi}$ :

$$
0=I\left(\sigma_{\mathrm{H}}\right)^{2}-c_{\xi}^{2} \Xi^{2} \sigma_{\mathrm{H}}^{4} I\left(\sigma_{\mathrm{H}}\right), \quad \therefore c_{\xi}^{2} \Xi^{2}=I\left(\sigma_{\mathrm{H}}\right) / \sigma_{\mathrm{H}}^{4} .
$$

Substituting this constant into the above, we obtain

$$
\xi^{\prime}(\sigma)=c_{\xi} \sigma^{4} \Xi^{2} \sqrt{\frac{\sigma^{2} H(\sigma) / \Delta(\sigma)}{\left(\sigma^{2} H(\sigma) G(\sigma)+I(\sigma)\right)\left(\sigma^{2} H(\sigma) G(\sigma)+I(\sigma)-I\left(\sigma_{\mathrm{H}}\right) \sigma^{4} / \sigma_{\mathrm{H}}^{4}\right)}},
$$

and the Lagrangian

$$
\begin{aligned}
\frac{\mathcal{L}_{\mathrm{KA} 4}}{T_{\mathrm{S}}} & =\frac{\left[\sigma^{2} H G+I\right]}{c_{\xi} \sigma^{4} \Xi^{2}} \xi^{\prime}(\sigma) \\
& =\sqrt{\frac{\left(\sigma^{2} H(\sigma) G(\sigma)+I(\sigma)\right) \sigma^{2} H(\sigma) / \Delta(\sigma)}{\sigma^{2} H(\sigma) G(\sigma)+I(\sigma)-I\left(\sigma_{\mathrm{H}}\right) \sigma^{4} / \sigma_{\mathrm{H}}^{4}}} .
\end{aligned}
$$

Action-The outer and the inner horizon are the smaller and larger solutions of $\Delta_{r}(r)=0$, respectively. The integral in the WDW patch is

$$
\begin{aligned}
& \frac{1}{T_{\mathrm{s}}} \int_{r_{-}}^{r_{+}} d \sigma \mathcal{L}_{\mathrm{KA} 4} \\
& \quad=\int_{r_{-}}^{r_{+}} d \sigma \sqrt{\frac{\sigma^{2} H(\sigma)\left(\sigma^{2} H(\sigma) G(\sigma)+I(\sigma)\right)}{\Delta(\sigma)\left(\sigma^{2} H(\sigma) G(\sigma)+I(\sigma)-I\left(\sigma_{\mathrm{H}}\right) \sigma^{4} / \sigma_{\mathrm{H}}^{4}\right)}} .
\end{aligned}
$$

The numerical calculation gives the results shown in Figs. 13, 14, 15, and 16.

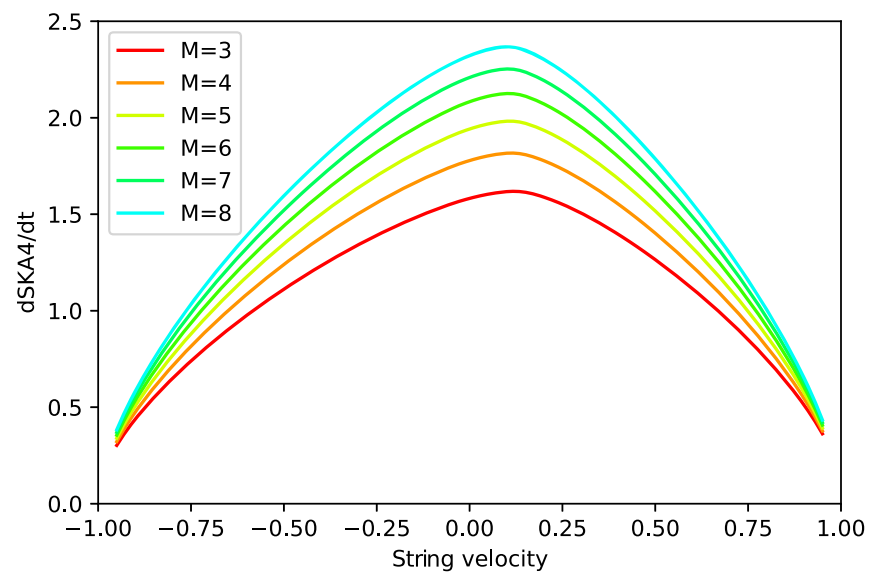

FIG. 13. Kerr-AdS $\mathrm{Ad}_{3+1}$ : Action growth vs string velocity $(a=0.1)$.
The first two figures, Figs. 13 and 14, show the string velocity dependence for different masses. Figure 13 is the result for $a=0.1$. The peak position is shifted to the right side. Figure 14 is, on the other hand, the result for a black hole with an angular momentum of the opposite direction

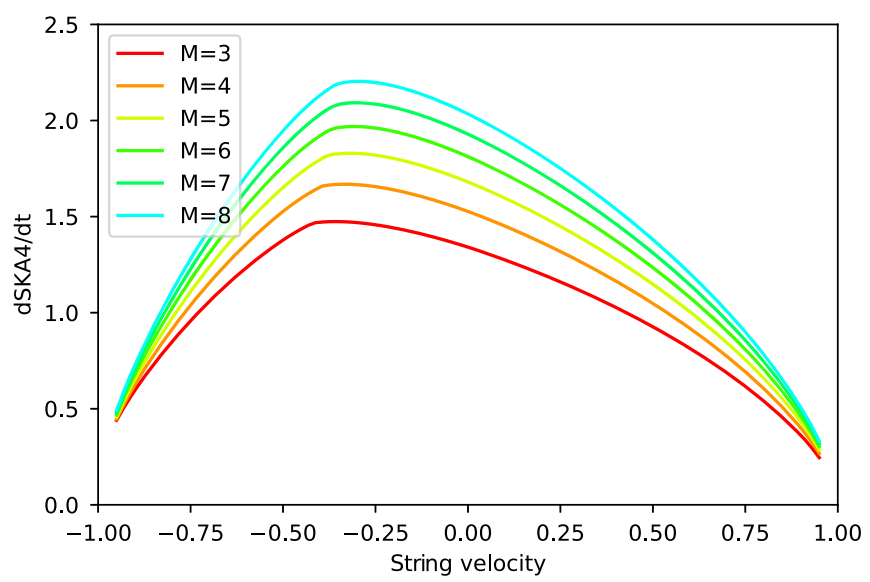

FIG. 14. Kerr-AdS $\mathrm{A}_{3+1}$ : Action growth vs string velocity $(a=-0.2)$.

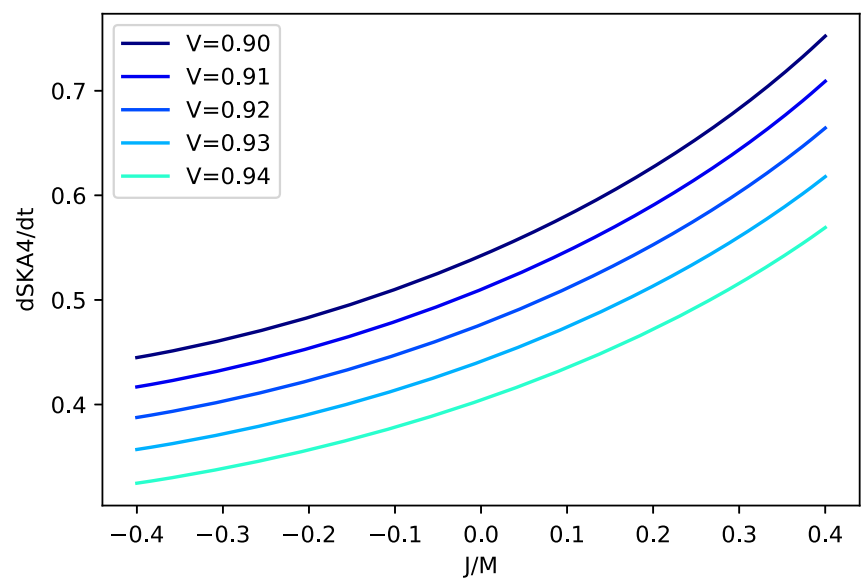

FIG. 15. Kerr-AdS ${ }_{3+1}$ : Action growth vs black hole $J / M$ $(m=5)$. 


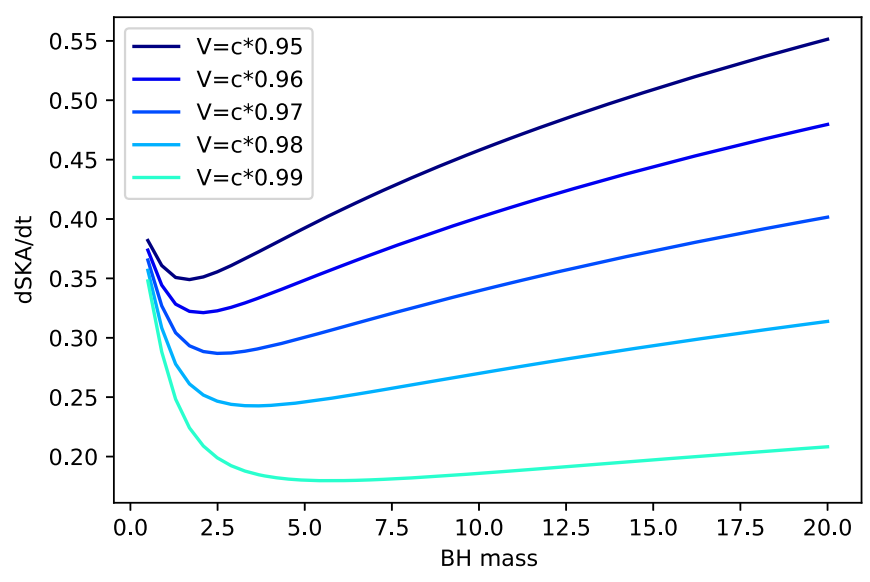

FIG. 16. Kerr-AdS ${ }_{3+1}$ : Action growth vs black hole mass $(a=0.1)$.

$a=-0.2$. The peak position is shifted to the other side. This behavior is consistent with the property that complexity is larger as the probe string motion slows down. That is, the effect takes the peak value when the relative velocity between the string and the black hole is zero. Not only that, but the peak value also decreases as the shift becomes larger, similar to the BTZ black hole case.

Figure 15 is the dependence of the angular momentum per black hole mass. This figure also shows that the effect on complexity is smaller as the string moves faster. And it also shows the tendency for complexity to be larger when the relative velocity is smaller because in this plot the string velocity is positive and the effect of complexity is larger in the positive region of the graph.

Figure 16 shows the mass dependence. As usual this is a increasing function of black hole mass. The faster string gives a small effect on complexity. But there is an unusual behavior in the small mass region where the extremum value appears.

\section{B. Five-dimensional Kerr-AdS black holes}

The $(4+1)$-dimensional Kerr-AdS black hole is described by (see Refs. [131-134])

$$
\begin{aligned}
d s_{\mathrm{KA} 5}^{2}= & -\frac{\Delta_{r}}{\rho^{2}}\left(d t-\frac{a \sin ^{2} \theta}{\Xi_{a}} d \phi_{1}-\frac{b \cos ^{2} \theta}{\Xi_{b}} d \phi_{2}\right)^{2}+\frac{\Delta_{\theta} \sin ^{2} \theta}{\rho^{2}}\left(a d t-\frac{r^{2}+a^{2}}{\Xi_{a}} d \phi_{1}\right)^{2}+\frac{\Delta_{\theta} \cos ^{2} \theta}{\rho^{2}}\left(b d t-\frac{r^{2}+b^{2}}{\Xi_{b}} d \phi_{2}\right)^{2} \\
& +\frac{\rho^{2}}{\Delta_{r}} d r^{2}+\frac{\rho^{2}}{\Delta_{\theta}} d \theta^{2}+\frac{1+r^{2}}{r^{2} \rho^{2}}\left(a b d t-\frac{b\left(r^{2}+a^{2}\right) \sin ^{2} \theta}{\Xi_{a}} d \phi_{1}-\frac{a\left(r^{2}+b^{2}\right) \cos ^{2} \theta}{\Xi_{b}} d \phi_{2}\right)^{2}
\end{aligned}
$$

where

$$
\begin{aligned}
\rho^{2}(r) & =r^{2}+a^{2} \cos ^{2} \theta+b^{2} \sin ^{2} \theta, \\
\Delta_{r}(r) & =\frac{1}{r^{2}}\left(r^{2}+a^{2}\right)\left(r^{2}+b^{2}\right)\left(r^{2}+1\right)-2 m, \\
\Delta_{\theta}(\theta) & =1-a^{2} \cos ^{2} \theta-b^{2} \sin ^{2} \theta, \\
\Xi_{a} & =1-a^{2}, \quad \Xi_{b}=1-b^{2} .
\end{aligned}
$$

The parameters here are related to the physical mass and the angular momentum as follows [133]:

$$
M=\frac{3 \pi m}{4 \Xi_{1} \Xi_{2}}, \quad J_{i}=\frac{\pi a_{i} m}{2 \Xi_{i}\left(1+r_{+}^{2}\right)} .
$$

We study the $a \neq 0$ case and the $b \neq 0$ case separately. These correspond to black holes rotating around different axes with coordinates $\phi_{1}$ and $\phi_{2}$. As before, we assume that the string moves in the great circle: $\theta=\pi / 2$.

\section{1. $a \neq 0$ case}

First we consider the case in which only $a$ is nonzero. In this case the string rotates around the same axis to the black hole:

$$
\begin{aligned}
d s_{\mathrm{KA} 5 \mathrm{a}}^{2}= & -\frac{\Delta_{r a}}{r^{2}}\left(d t-\frac{a}{\Xi_{a}} d \phi_{1}\right)^{2}+\frac{r^{2}}{\Delta_{r a}} d r^{2} \\
& +\frac{1}{r^{2}}\left(a d t-\frac{r^{2}+a^{2}}{\Xi_{a}} d \phi_{1}\right)^{2}, \\
\Delta_{r a}= & \left(r^{2}+a^{2}\right)\left(r^{2}+1\right)-2 m .
\end{aligned}
$$

This looks like the same form as the four-dimensional Kerr-AdS case except that the function $\Delta_{r}(r)$ is replaced by $\Delta_{r a}(r)$ (the second term does not depend on $r$ ). We need the same shift (35) to relate the velocity parameter $v$ to the string velocity $V: v=V-a$. The parametrization of the string world sheet is

$$
t=\tau, \quad r=\sigma, \quad \Phi=V \tau+\xi(\sigma) .
$$

EOM and its solution-The calculation of the induced metric and the NG action are performed in the same way as the Kerr- $\mathrm{AdS}_{3+1}$ case. So the Lagrangian is the same form as Eq. (48) except that $\Delta(r)$ is replaced by $\Delta_{a}$ :

$$
\frac{\mathcal{L}_{\mathrm{KA} 5 \mathrm{a}}}{T_{\mathrm{S}}}=\sqrt{\frac{\left(\sigma^{2} H(\sigma) G(\sigma)+I(\sigma)\right) \sigma^{2} H(\sigma) / \Delta_{a}(\sigma)}{\sigma^{2} H(\sigma) G(\sigma)+I(\sigma)-I\left(\sigma_{\mathrm{H}}\right) \sigma^{4} / \sigma_{\mathrm{H}}^{4}}},
$$


where

$$
\begin{aligned}
\Delta_{a}(\sigma):=\left(\sigma^{2}+a^{2}\right)\left(\sigma^{2}+1\right)-2 m, \\
F(\sigma):=\Delta_{a}(\sigma)-\left(\sigma^{2}+a^{2}\right), \\
G(\sigma):=\left(\sigma^{2}+a^{2}\right)^{2}-a^{2} \Delta_{a}(\sigma), \\
H(\sigma):=\frac{\Delta_{a}(\sigma)}{\sigma}-\frac{a^{2}}{\sigma}-2 \frac{a v}{\Xi_{a}}-v^{2} \frac{G(\sigma)}{\sigma^{2} \Xi^{2}}, \\
I(\sigma):=\left(a F(\sigma)+v \frac{G}{\Xi}\right)^{2} .
\end{aligned}
$$

The inner and outer horizons are determined by

$$
\Delta_{r a}\left(r_{ \pm}\right)=0
$$

Action-The action integrated over the WDW patch is

$\frac{d S_{\mathrm{NG}}}{d t}=\int_{r_{-}}^{r_{+}} d \sigma \sqrt{\frac{\left(\sigma^{2} H(\sigma) G(\sigma)+I(\sigma)\right) \sigma^{2} H(\sigma) / \Delta_{a}(\sigma)}{\sigma^{2} H(\sigma) G(\sigma)+I(\sigma)-I\left(\sigma_{\mathrm{H}}\right) \sigma^{4} / \sigma_{\mathrm{H}}^{4}}}$.

This integration is performed by the numerical calculation. The result shows the string velocity dependence, the black hole angular momentum dependence and the mass dependence.

The velocity dependence is shown in Figs. 17 and 18. We can see as usual that the effect on complexity is larger for a larger mass and the effect is at maximum when the string is stationary. But the difference for different values of parameter $a$ disappears.

The angular momentum per unit mass $(a=J / M)$ dependence is shown in Fig. 19. Similar to the velocity dependence, a sharp slope tends to disappear. There is a

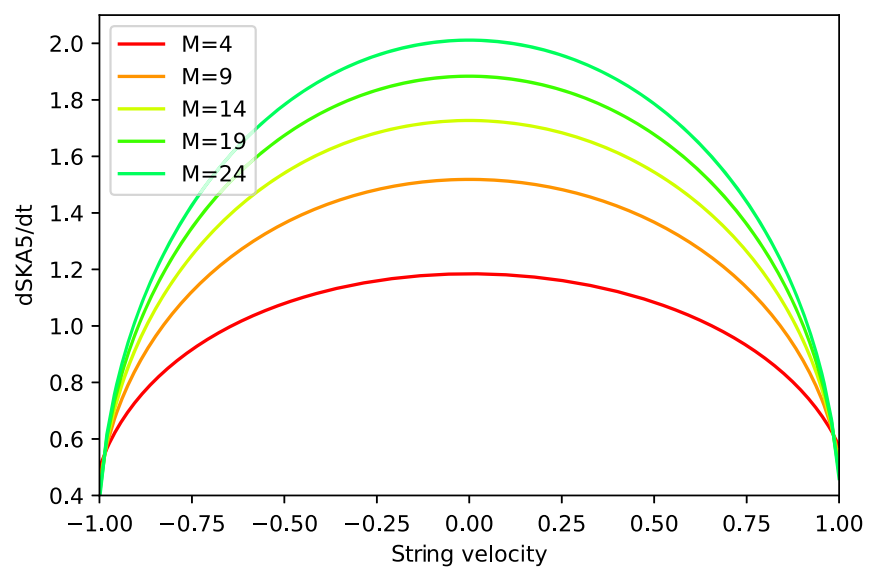

FIG. 17. Kerr-AdS $4+1$ : Action growth vs velocity $(a=0.1)$.

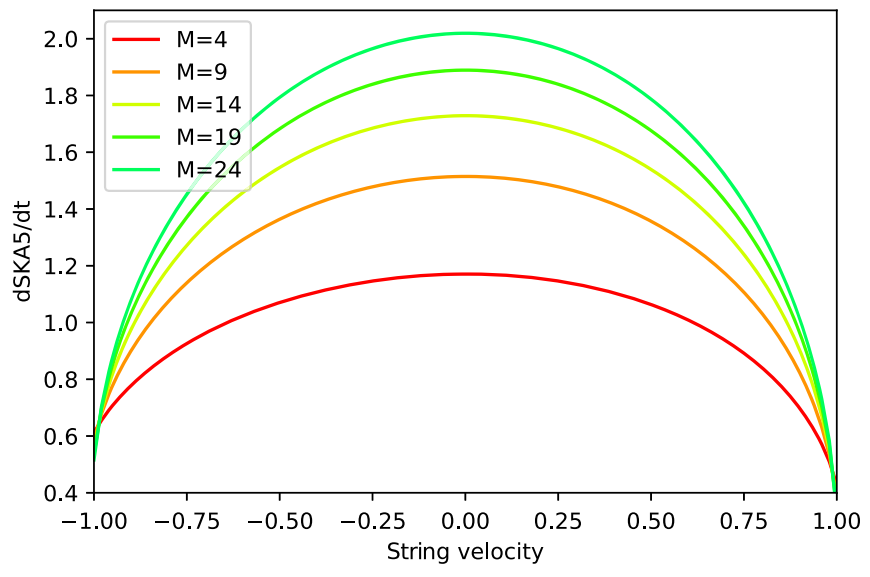

FIG. 18. Kerr-AdS $\mathrm{A}_{4+1}$ : Action growth vs $m(a=-0.2)$.

universal behavior - the effect of the string is small when the relative velocity is large.

The mass dependence is shown in Fig. 20. Basically the effect increases according to the black hole mass, but this

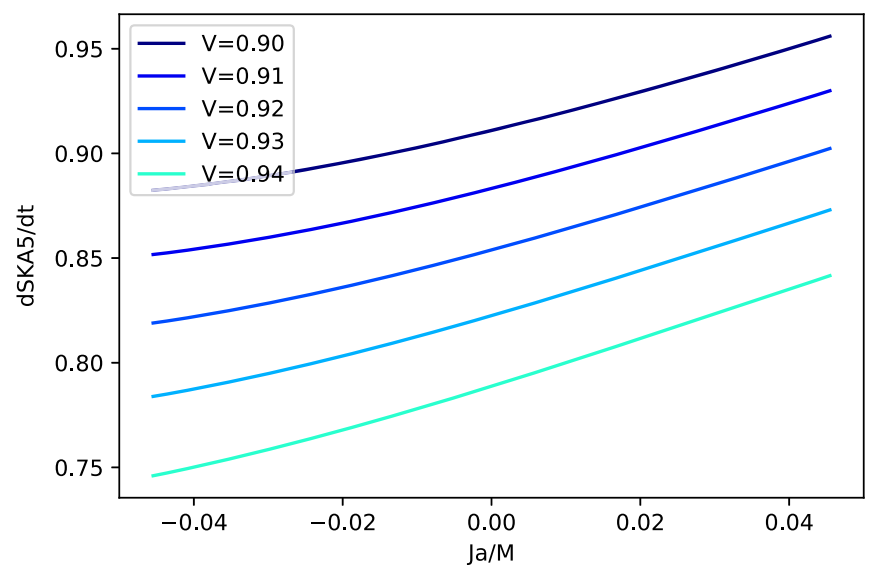

FIG. 19. Kerr-AdS ${ }_{4+1}$ : Action growth vs black hole $J_{a} / M$ $(m=5)$.

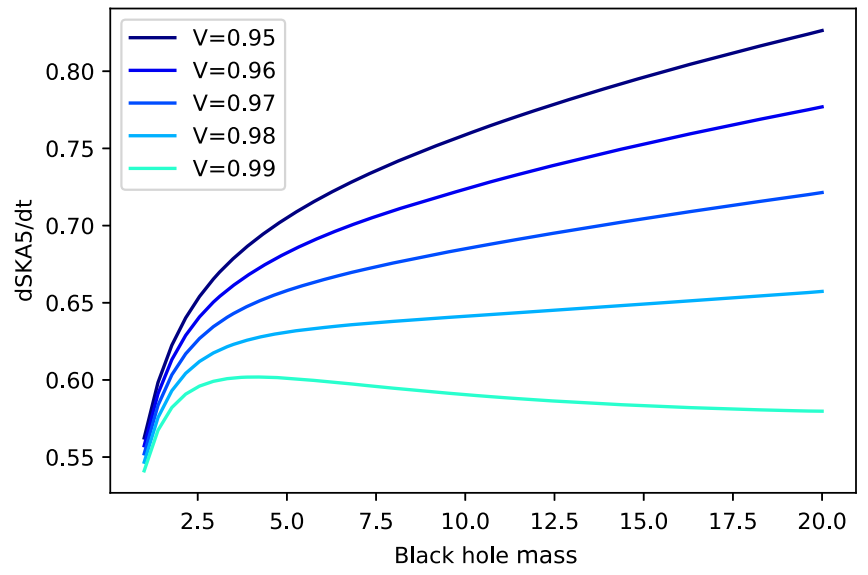

FIG. 20. Kerr-AdS ${ }_{4+1}$ : Action growth vs black hole mass $(a=0.1)$. 
changes to the decreasing function once the string velocity exceeds the threshold (it is almost light speed, $\approx 0.98 c$ ).

\section{2. $b \neq 0$ case}

Next let us consider the $b \neq 0$ case. In this case the string moves in the axis to the black hole. The metric becomes

$$
\begin{aligned}
d s_{\mathrm{KA} 5 \mathrm{~b}}^{2}= & -\frac{\Delta_{r b}}{r^{2}+b^{2}} d t^{2}+\frac{r^{2}+b^{2}}{\Delta_{r b}} d r^{2} \\
& +\frac{r^{4}\left(1-b^{2}\right)+b^{2} r^{2}\left(1+r^{2}\right)}{r^{2}+b^{2}} d \phi_{1}^{2}, \\
\Delta_{r b}= & \left(r^{2}+b^{2}\right)\left(r^{2}+1\right)-2 m, \\
= & -\left(r^{2}+1-\frac{2 m}{r^{2}+b^{2}}\right) d t^{2} \\
& +\left(r^{2}+1-\frac{2 m}{r^{2}+b^{2}}\right)^{-1} d r^{2}+r^{2} d \phi_{1}^{2} .
\end{aligned}
$$

Since $\Xi_{a}=1$, from Eq. (51) the angular momentum per black hole mass is

$$
\frac{J_{b}}{M}=\frac{2}{3} \frac{b}{1+r_{+}^{2}}
$$

We choose the same parametrization as before:

$$
\tau=t, \quad r=\sigma, \quad \phi_{1}=v \tau+\xi(\sigma) .
$$

Note that the above metric is already in the AdS form. Then one does not need to shift the velocity (35) to relate the string velocity to the parameter $v(V=v)$. The induced metric is

$$
\begin{aligned}
d s_{\mathrm{KA} \text { b }: \text { ind }}^{2}= & -f(\sigma) d \tau^{2}+\frac{d \sigma^{2}}{f(\sigma)}+\sigma^{2}\left(v d \tau+\xi^{\prime} d \sigma\right)^{2} \\
f(\sigma):= & 1+\sigma^{2}-\frac{2 m}{\sigma^{2}+b^{2}} \\
= & -\left(f(\sigma)-v^{2} \sigma^{2}\right) d \tau^{2}+\left(\frac{1}{f(\sigma)}+\sigma^{2} \xi^{\prime 2}\right) d \sigma^{2} \\
& +2 v \sigma^{2} \xi^{\prime} d \tau d \sigma .
\end{aligned}
$$

EOM and its solution-This induced metric is the same form as the $\operatorname{AdS}_{n+1}$ (4) case except that the function $f(\sigma)$ is replaced. Then the equation of motion is now obtained only by replacing the old $f(\sigma)$ with the new one,

$$
\begin{aligned}
\xi^{\prime}(\sigma) & =\frac{c_{\xi}}{\sigma^{2} f(\sigma)} \frac{\mathcal{L}_{\mathrm{KA} 5 \mathrm{~b}}}{T_{\mathrm{s}}}=\frac{c_{\xi}}{\sigma^{2} f(\sigma)} \sqrt{\frac{\sigma^{2} f(\sigma)-v^{2} \sigma^{4}}{\sigma^{2} f(\sigma)-c_{\xi}^{2}}}, \\
f(\sigma) & =1+\sigma^{2}-\frac{2 m}{\sigma^{2}+b^{2}} .
\end{aligned}
$$

The reality condition in the square root should be imposed. The numerator is

$$
\begin{aligned}
0 & =1+\left(1-v^{2}\right) \sigma^{2}-2 m /\left(\sigma^{2}+b^{2}\right) \\
& \Rightarrow\left(1-v^{2}\right) \sigma^{4}+\left(1+\left(1-v^{2}\right) b^{2}\right) \sigma^{2}+b^{2}-2 m=0 .
\end{aligned}
$$

Since $\quad D=\left(1+\left(1-v^{2}\right) b^{2}\right)^{2}+4\left(1-v^{2}\right)\left(2 m-b^{2}\right)=$ $\left(1-\left(1-v^{2}\right) b^{2}\right)^{2}+8 m\left(1-v^{2}\right)>0$, this equation certainly has real solutions. $\sigma_{\mathrm{H}}$ denotes a positive one of them:

$$
\begin{aligned}
\sigma_{\mathrm{H}}^{2}= & -\frac{1+\left(1-v^{2}\right) b^{2}}{2} \\
& +\frac{1}{2} \sqrt{\left(1-\left(1-v^{2}\right) b^{2}\right)^{2}+8 m\left(1-v^{2}\right)} .
\end{aligned}
$$

From the condition for the denominator, the constant $c_{\xi}$ is determined as $c_{\xi}^{2}=\sigma_{\mathrm{H}}^{2} f\left(\sigma_{\mathrm{H}}\right)$. The Lagrangian becomes

$$
\begin{aligned}
\frac{\mathcal{L}_{\mathrm{KA} 5 \mathrm{~b}}}{T_{\mathrm{S}}} & =\sqrt{\frac{\sigma^{2} f(\sigma)-v^{2} \sigma^{4}}{\sigma^{2} f(\sigma)-\sigma_{\mathrm{H}}^{2} f\left(\sigma_{\mathrm{H}}\right)}} \\
& =\sigma \sqrt{\frac{\left(1-v^{2}\right)\left(\sigma^{2}+\sigma_{\mathrm{H}}^{2}+b^{2}\right)+1}{\left(\sigma^{2}+b^{2}\right)\left(\sigma^{2}+\sigma_{\mathrm{H}}^{2}+1\right)-2 m b^{2} /\left(\sigma_{\mathrm{H}}^{2}+b^{2}\right)}} .
\end{aligned}
$$

The horizon is determined by

$$
\begin{aligned}
\Delta_{r b}(r) & =0 \\
\therefore r_{\mathrm{h}} & =\left(-\frac{1+b^{2}}{2}+\frac{1}{2} \sqrt{\left(1-b^{2}\right)^{2}+8 m}\right)^{1 / 2} .
\end{aligned}
$$

Action-Then the development of the NG action obtained by integrating over the WDW patch is

$$
\begin{aligned}
& \frac{d S_{\mathrm{NG}}}{d t} \\
& =\int_{0}^{r_{\mathrm{h}}} d \sigma \sigma \sqrt{\frac{\left(1-v^{2}\right)\left(\sigma^{2}+\sigma_{\mathrm{H}}^{2}+b^{2}\right)+1}{\left(\sigma^{2}+b^{2}\right)\left(\sigma^{2}+\sigma_{\mathrm{H}}^{2}+1\right)-2 m b^{2} /\left(\sigma_{\mathrm{H}}^{2}+b^{2}\right)}} \\
& =\frac{1}{2} \int_{0}^{r_{\mathrm{h}}^{2}} d \sigma^{2} \sqrt{\frac{\left(1-v^{2}\right)\left(\sigma^{2}+\sigma_{\mathrm{H}}^{2}+b^{2}\right)+1}{\left(\sigma^{2}+b^{2}\right)\left(\sigma^{2}+\sigma_{\mathrm{H}}^{2}+1\right)-2 m b^{2} /\left(\sigma_{\mathrm{H}}^{2}+b^{2}\right)}} .
\end{aligned}
$$

Figures 21, 22, 23, and 24 show the result of the numerical calculation.

The velocity dependence is shown in Figs. 21 and 22. As usual, the effect on complexity takes the extremum when the string is stationary. There is abnormal behavior in the 


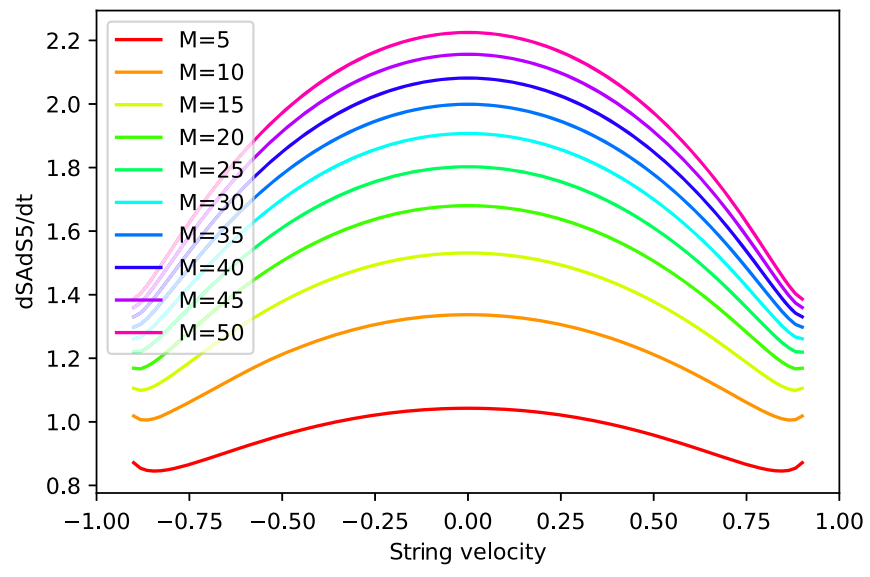

FIG. 21. Kerr-AdS $\mathrm{A}_{4+1}$ : Action growth vs velocity $(b=0.1)$.

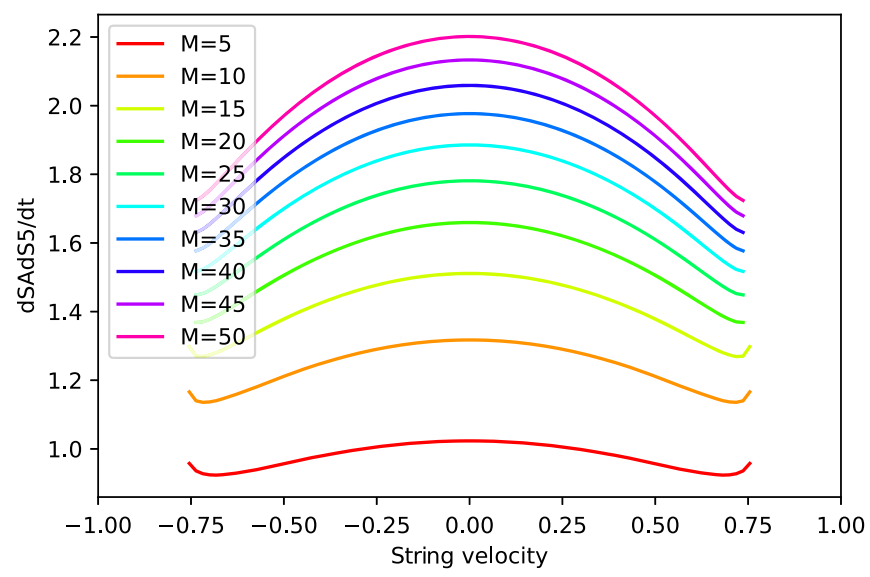

FIG. 22. Kerr-AdS $\mathrm{A}_{4+1}$ : Action growth vs velocity $(b=-0.2)$.

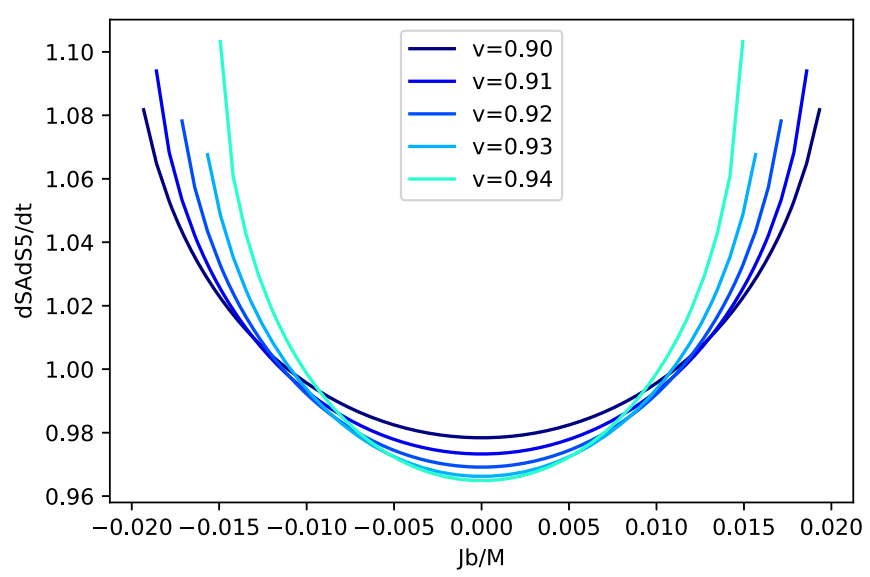

FIG. 23. Kerr-AdS $4+1$ : Action growth vs velocity $(b=0.1)$.

vicinity of light speed. It tends to increase a bit in the maximum of the velocity. Because of the reality condition the velocity cannot reach light speed. This restricted region becomes narrow with an increase in the absolute value of $b$. This is a new phenomena that we found.

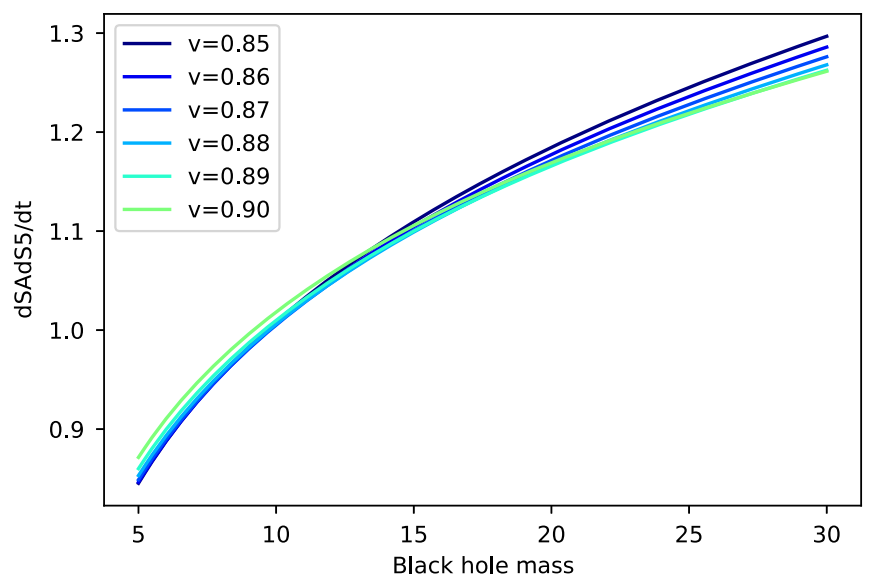

FIG. 24. Kerr-AdS $\mathrm{A}_{4+1}$ : Action growth vs black hole mass $(b=0.1)$.

The dependence on the angular momentum is shown in Fig. 23. Since the string rotates in a different axis, the relative velocity never becomes zero. Then this behaves very differently than the previous ones.

Figure 24 shows the mass dependence. The difference between different velocities ceases, and there is no extremum point in this case. While in the small mass region fast strings give a large effect, in the large mass region the slower strings have a larger effect, as usual.

\section{DISCUSSION}

\section{A. Summary}

We have seen the effect of the probe string in BTZ, $\mathrm{AdS}_{3+1}, \mathrm{AdS}_{4+1}, \mathrm{AdS}_{5+1}$, and Kerr-AdS black hole spacetime. The previous work [126] revealed the effect of the probe string in different masses and string velocities. We could confirm this result and that is a universal behavior in more broad type of black holes. More specifically, complexity shows different behavior according to the string velocity, the black hole mass, and the spacetime dimension. Let us summarize these factors and their physical interpretation here.

Velocity dependence-A stationary string gives the maximal complexity growth. Complexity decreases as the probe string moves faster. This seems to contradict the physical intuition because complexity measures how difficult it is to create the target state from the initial state, which is usually stationary. The same phenomenon was also found in the previous work [126]. Then we can conclude that this is a universal property of complexity.

The position of the maximum is shifted in the rotating black hole. This is thought to be derived from the relative velocity between the string velocity and the black hole angular momentum. That is, the effect on complexity is larger when the relative velocity is smaller. The maximum 
value also decreases as the maximum point moves from the center by this shift. Near light speed there is also an interesting phenomena in the mass dependence, as stated below.

Let us note that the universal property of complexity stated above does not stem from the time delation of relativistic phenomena. Figures 7, 8, 13, and 14 show the peak position shifts according the relative velocity between the black hole and the probe string. Since we calculated the NG action on the rest flame, the peak locates at $v=0$ if this behavior stems from the time dilation. We can also see that if this behavior derives from the Lorentz factor $\sqrt{1-v^{2}}$, it does not behave linearly as the BTZ cases (Figs. 7 and 8).

Mass dependence-Complexity basically tends to increase according the mass. This can be thought that this is because complexity defines how complex the physical system is.

A remarkable phenomenon occurs in the vicinity of light speed. In the lower dimension, $\operatorname{AdS}_{3+1}$ and $\operatorname{AdS}_{4+1}$, the dependence on black hole mass has the maximum point for near light speed strings. That maximum disappears for higher dimension, as shown in the $\mathrm{AdS}_{5+1}$-dimensional case (Fig. 6).

In lower dimension, $\mathrm{AdS}_{n+1 \leq 5}$, the mass dependence can be a decreasing function of mass for a near light speed string.

Dimensionality dependence-As the spacetime dimension becomes higher, the peak of the dependence on the string velocity becomes smooth. Specifically, in the threedimensional case, the velocity dependence in BTZ black holes forms a broken line. As the dimensionality becomes higher, this slope tends to become gentle.

We can conclude that the effect of the probe string becomes insensitive in higher dimensions. It can be intuitively explained as follows. Although the NambuGoto action is proportional to the two-dimensional world volume in whole spacetime, we restrict the motion of the string in the $S^{1}$ subspace of a specific plane. In order to remove this restriction, we investigate in Sec. IV B 2 the case where string moves around a different axis to the black hole angular momentum. As expected, a new phenomena was found. That is, the dependence on the string velocity does not decrease near light speed (see Figs. 21 and 22). Furthermore, the difference of the dependence on mass in various string velocities disappears in this case (see Fig. 24).

Maximum value-According to the results in Sec. II (Figs. 1, 3, and 5), the plots of the velocity dependence not only become smoother, but their maximum value also looks to decrease. Let us confirm that this behavior is universal. We focus on $\mathrm{AdS}_{n+1}$ black holes. We know already that the effect of the string is maximum when the string velocity is zero. For $v=0$ the Lagrangian (13) is unity. The integral depends only on the horizon:

$$
\frac{\mathcal{L}_{\mathrm{AdS}(n+1)}}{T_{\mathrm{s}}}=r_{\mathrm{h}} .
$$

The horizon $r_{\mathrm{h}}$ is determined by [see the metric function (2)]

$$
\begin{aligned}
0 & =f(r)=1-\frac{16 \pi}{(n-1) \Omega_{n-1}} \frac{M}{r^{n-2}}+r^{2} \\
& \Rightarrow r^{n}+r^{n-2}-\frac{8 \pi^{-n / 2+1} \Gamma(n / 2)}{n-1} M=0 .
\end{aligned}
$$

The maximum value of the NG action in diverse dimensions is plotted in Fig. 25.

There is a difficulty in the charged case, as I explain later of Eq. (72). But only the maximum value can be obtained in the same way as the uncharged case. We focus on the extremal black holes. The Lagrangian is again unity, and the action is equal to the horizon. This horizon is determined in the same way by

$$
r^{n}+r^{n-2}-\frac{4 \pi^{-n / 2+1} \Gamma(n / 2)}{n-1}\left(2 M-\frac{Q^{2}}{r}\right)=0 .
$$

Given a charge, the extremal mass is determined by setting the minimum value of the left-hand side of Eq. (70) at zero. The dimensionality dependence of the maximum value of the NG action for extremal black holes is plotted in Fig. 26. In this plot we see that the maximum value decreases at lower dimensions. Furthermore, the minimum point approaches ten dimensions for sufficiently large charges.

\section{B. Future direction}

In this work we considered the effect of the probe string. That corresponds to the introduction of a kind of nonlocal operator-a Wilson loop. We first expect the generalization of the dimension. Several higherdimensional local operators can be added. Specifically,

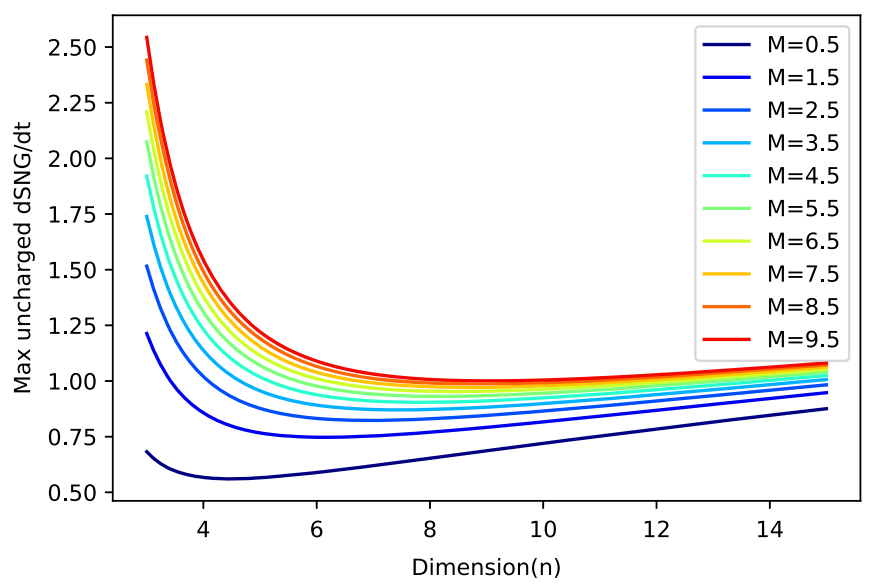

FIG. 25. Maximum of the action growth in diverse dimensions (uncharged case). 


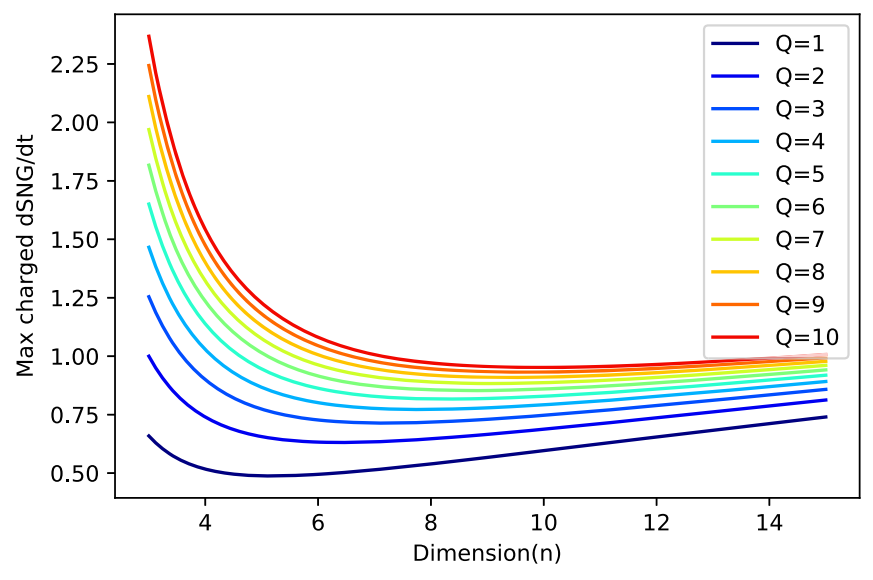

FIG. 26. Maximum of the action growth in diverse dimensions (charged case).

the codimension local operator, the interface, is an interesting object. This local operator realized in a system consisting of two kinds of branes-D3 and D5. Since complexity is known to have a nonlocal property, it must be useful to use such operators to study the property of complexity. Some interesting properties of the nonlocal operators in BTZ black holes are already found in Ref. [117], and complexity growth of the defect theory is studied in Ref. [135]. One suggestion is to study the effect of these nonlocal operators on the diverse kinds of black holes.

In Eq. (1) we restrict the case to uncharged AdS black holes. The growth of the Einstein-Hilbert action for the charged case is studied in Ref. [136]. I would like to study the nonlocal operators in these kinds of black holes. The adding of the charge is important future work since it is related to checking whether the complexity growth satisfies the Lloyd bound [73,113]. But in this case a difficulty occurs. The metric function in this case is

$$
\begin{array}{r}
d s_{\mathrm{AdS}_{n+1}}^{2}=-f(r) d t^{2}+\frac{d r^{2}}{f(r)}+r^{2} d \Omega_{n-1}, \\
f(r)=1-\frac{8 \pi}{(n-1) \Omega_{n-1}}\left(\frac{2 G M}{r^{n-2}}-\frac{G Q^{2}}{r^{n-1}}\right)+\frac{r^{2}}{\ell_{\mathrm{AdS}}^{2}} \\
=1-\frac{r_{\mathrm{m}}^{n-2}}{r^{n-2}}+\frac{r^{2}}{\ell_{\mathrm{AdS}}^{2}}, \\
r_{\mathrm{m}}^{n-2}:=\frac{16 \pi G M}{(n-1) \Omega_{n-1}}, \quad r_{\mathrm{q}}^{n-1}:=\frac{8 \pi G Q^{2}}{(n-1) \Omega_{n-1}} .
\end{array}
$$

The condition for the numerator of Eq. (10) is changed by adding a new term,

$$
\left(1-v^{2}\right) \sigma^{n}+\sigma^{n-2}-r_{\mathrm{m}}^{n-2}+r_{\mathrm{q}}^{n-1} / \sigma=0 .
$$

The left-hand side of this equation is no longer monotonically increasing. If this function has real solutions, there are two solutions at least (including multiple ones). So the same procedure cannot be used since we need to determine the constant $c_{\xi}$ in the denominator of Eq. (9) using the unique solution of the above equation.

\section{ACKNOWLEDGMENTS}

I would like to thank Satoshi Yamaguchi, UESTC, and KEK members and people having discussions at the 73th Physical Society of Japan Annual Meeting for helping with my research.
[1] T. J. Osborne, Hamiltonian complexity, Rep. Prog. Phys. 75, 022001 (2012).

[2] Sevag Gharibian, Yichen Huang, Zeph Landau, and Seung Woo Shin, Quantum Hamiltonian complexity, Found. Trends Theor. Comput. Sci. 10, 159 (2015).

[3] G. Dvali, C. Gomez, D. Lust, Y. Omar, and B. Richter, Universality of black hole quantum computing, Fortschr. Phys. 65, 1600111 (2017).

[4] B. Swingle, G. Bentsen, M. Schleier-Smith, and P. Hayden, Measuring the scrambling of quantum information, Phys. Rev. A 94, 040302 (2016).

[5] K. Hashimoto, N. Iizuka, and S. Sugishita, Time evolution of complexity in Abelian gauge theories, Phys. Rev. D 96, 126001 (2017).

[6] J. Watrous, Quantum computational complexity, arXiv: 0804.3401.
[7] N. Bao and J. Liu, Quantum complexity and the virial theorem, J. High Energy Phys. 08 (2018) 144.

[8] S. Arora and B. Barak, Computational Complexity: A Modern Approach, 1st ed. (Cambridge University Press, New York, 2009).

[9] C. Moore and S. Mertens, The Nature of Computation (Oxford University Press, New York, 2011).

[10] S. R. Coleman, J. Preskill, and F. Wilczek, Quantum hair on black holes, Nucl. Phys. B378, 175 (1992).

[11] J. Preskill, Do black holes destroy information?, in Proceedings of the International Symposium on Black Holes, Membranes, Wormholes and Superstrings Woodlands, Texas, 1992, edited by K. Sunny and D. V. Nanopoulos (World Scientific, Singapore, 1992), p. 22.

[12] S. B. Giddings, Comments on information loss and remnants, Phys. Rev. D 49, 4078 (1994). 
[13] J. G. Russo, The information problem in black hole evaporation: Old and recent results, in Beyond General Relativity: Proceedings of the 2004 Spanish Relativity Meeting (ERE2004), edited by N. Alonso-Alberca, E. Alvarez, T. Ortin, and M. A. Vazquez-Mozo (Universidad Autónoma de Madrid, Spain, 2007), p. 218.

[14] Y. Sekino and L. Susskind, Fast scramblers, J. High Energy Phys. 10 (2008) 065.

[15] D. R. Terno, Black hole information problem and quantum gravity, AIP Conf. Proc. 1196, 284 (2009).

[16] T. Hartman and J. Maldacena, Time evolution of entanglement entropy from black hole interiors, J. High Energy Phys. 05 (2013) 014.

[17] K. Bradler and C. Adami, The capacity of black holes to transmit quantum information, J. High Energy Phys. 05 (2014) 095.

[18] J. Polchinski, The black hole information problem, in Proceedings of the 2015 Theoretical Advanced Study Institute in Elementary Particle Physics (TASI 2015), Boulder, 2015, edited by J. Polchinski, P. Vieira, and O. DeWolfe (World Scientific, Singapore, 2017), pp. 353-397.

[19] D. Marolf, The black hole information problem: Past, present, and future, Rep. Prog. Phys. 80, 092001 (2017).

[20] L. Susskind, Singularities, firewalls, and complementarity, arXiv:1208.3445.

[21] A. Almheiri, D. Marolf, J. Polchinski, and J. Sully, Black holes: Complementarity or firewalls?, J. High Energy Phys. 02 (2013) 062.

[22] H. Stoltenberg and A. Albrecht, No firewalls or information problem for black holes entangled with large systems, Phys. Rev. D 91, 024004 (2015).

[23] P. Hayden and J. Preskill, Black holes as mirrors: Quantum information in random subsystems, J. High Energy Phys. 09 (2007) 120.

[24] D. Harlow and P. Hayden, Quantum computation vs firewalls, J. High Energy Phys. 06 (2013) 085.

[25] L. Susskind, Entanglement is not enough, Fortschr. Phys. 64, 49 (2016).

[26] R. B. Mann, Black Holes: Thermodynamics, Information, and Firewall, SpringerBriefs in Physics (Springer, New York, 2015).

[27] J. L. F. Barbon and E. Rabinovici, Holographic complexity and spacetime singularities, J. High Energy Phys. 01 (2016) 084.

[28] J. L. F. Barbon and J. Martin-Garcia, Holographic complexity of cold hyperbolic black holes, J. High Energy Phys. 11 (2015) 181.

[29] J. Couch, W. Fischler, and P. H. Nguyen, Noether charge, black hole volume, and complexity, J. High Energy Phys. 03 (2017) 119.

[30] Z. Fu, D. Marolf, and E. Mefford, Time-independent wormholes, J. High Energy Phys. 12 (2016) 021.

[31] Y. Zhao, Complexity, boost symmetry, and firewalls, Phys. Rev. D 98, 086011 (2018).

[32] J. Maldacena and L. Susskind, Cool horizons for entangled black holes, Fortschr. Phys. 61, 781 (2013).

[33] D. Stanford and L. Susskind, Complexity and shock wave geometries, Phys. Rev. D 90, 126007 (2014).
[34] L. Susskind, Computational complexity and black hole horizons, Fortschr. Phys. 64, 24 (2016).

[35] L. Susskind, The typical-state paradox: Diagnosing horizons with complexity, Fortschr. Phys. 64, 84 (2016).

[36] D. A. Roberts and B. Yoshida, Chaos and complexity by design, J. High Energy Phys. 04 (2017) 121.

[37] A. R. Brown, L. Susskind, and Y. Zhao, Quantum complexity and negative curvature, Phys. Rev. D 95, 045010 (2017).

[38] W. Cottrell and M. Montero, Complexity is simple!, J. High Energy Phys. 02 (2018) 039.

[39] A. P. Reynolds and S. F. Ross, Complexity of the AdS soliton, Classical Quantum Gravity 35, 095006 (2018).

[40] R. Q. Yang, Complexity for quantum field theory states and applications to thermofield double states, Phys. Rev. D 97, 066004 (2018).

[41] M. Kord Zangeneh, Y. C. Ong, and B. Wang, Entanglement entropy and complexity for one-dimensional holographic superconductors, Phys. Lett. B 771, 235 (2017).

[42] L. Susskind, Black holes and complexity classes, arXiv: 1802.02175.

[43] R. Khan, C. Krishnan, and S. Sharma, Circuit complexity in fermionic field theory, Phys. Rev. D 98, 126001 (2018).

[44] V. Vanchurin, Dual field theories of quantum computation, J. High Energy Phys. 06 (2016) 001.

[45] S. Chapman, M. P. Heller, H. Marrochio, and F. Pastawski, Towards Complexity for Quantum Field Theory States, Phys. Rev. Lett. 120, 121602 (2018).

[46] J. Jiang, J. Shan, and J. Yang, Circuit complexity for free fermion with a mass quench, arXiv:1810.00537.

[47] J. Molina-Vilaplana and A. Del Campo, Complexity functionals and complexity growth limits in continuous MERA circuits, J. High Energy Phys. 08 (2018) 012.

[48] A. Bhattacharyya, P. Caputa, S. R. Das, N. Kundu, M. Miyaji, and T. Takayanagi, Path-integral complexity for perturbed CFTs, J. High Energy Phys. 07 (2018) 086.

[49] A. P. Reynolds, Exploring holographic chaos and complexity, Ph.D. thesis, Durham University, 2018.

[50] G. Evenbly and G. Vidal, Tensor Network Renormalization, Phys. Rev. Lett. 115, 180405 (2015).

[51] A. May, Tensor networks for dynamic spacetimes, J. High Energy Phys. 06 (2017) 118.

[52] A. Bhattacharyya, Z.-S. Gao, L.-Y. Hung, and S.-N. Liu, Exploring the tensor networks/AdS correspondence, J. High Energy Phys. 08 (2016) 086.

[53] N. Bao, C. Cao, S. M. Carroll, and A. Chatwin-Davies, de Sitter space as a tensor network: Cosmic no-hair, complementarity, and complexity, Phys. Rev. D 96, 123536 (2017).

[54] A. May, Tensor networks for dynamic spacetimes, master's thesis, University of British Columbia, 2017.

[55] P. Caputa, N. Kundu, M. Miyaji, T. Takayanagi, and K. Watanabe, Liouville action as path-integral complexity: From continuous tensor networks to AdS/CFT, J. High Energy Phys. 11 (2017) 097.

[56] A. Peach and S.F. Ross, Tensor network models of multiboundary wormholes, Classical Quantum Gravity 34, 105011 (2017). 
[57] L. Susskind and Y. Zhao, Switchbacks and the bridge to nowhere, arXiv:1408.2823.

[58] M. A. Nielsen, A geometric approach to quantum circuit lower bounds, arXiv:quant-ph/0502070.

[59] M. A. Nielsen, M. R. Dowling, M. Gu, and A. C. Doherty, Quantum computation as geometry, Science 311, 1133 (2006).

[60] M. R. Dowling and M.A. Nielsen, The geometry of quantum computation, arXiv:quant-ph/0701004.

[61] R. Jefferson and R. C. Myers, Circuit complexity in quantum field theory, J. High Energy Phys. 10 (2017) 107.

[62] R. Q. Yang, Y. S. An, C. Niu, C. Y. Zhang, and K. Y. Kim, Axiomatic complexity in quantum field theory and its applications, arXiv:1803.01797.

[63] L. Hackl and R. C. Myers, Circuit complexity for free fermions, J. High Energy Phys. 07 (2018) 139.

[64] J. M. Maldacena, The large $N$ limit of superconformal field theories and supergravity, Int. J. Theor. Phys. 38, 1113 (1999); Adv. Theor. Math. Phys. 2, 231 (1998).

[65] M. Alishahiha, Holographic complexity, Phys. Rev. D 92, 126009 (2015).

[66] W. Chemissany and T. J. Osborne, Holographic fluctuations and the principle of minimal complexity, J. High Energy Phys. 12 (2016) 055.

[67] X. H. Ge and B. Wang, Quantum computational complexity, Einstein's equations and accelerated expansion of the Universe, J. Cosmol. Astropart. Phys. 02 (2018) 047.

[68] B. Czech, Einstein Equations from Varying Complexity, Phys. Rev. Lett. 120, 031601 (2018).

[69] S. A. Hosseini Mansoori and M. M. Qaemmaqami, Complexity growth, butterfly velocity and black hole thermodynamics, arXiv:1711.09749.

[70] M. Moosa, Divergences in the rate of complexification, Phys. Rev. D 97, 106016 (2018).

[71] R. Auzzi, S. Baiguera, and G. Nardelli, Volume and complexity for warped AdS black holes, J. High Energy Phys. 06 (2018) 063.

[72] A. R. Brown, D. A. Roberts, L. Susskind, B. Swingle, and Y. Zhao, Holographic Complexity Equals Bulk Action?, Phys. Rev. Lett. 116, 191301 (2016).

[73] A. R. Brown, D. A. Roberts, L. Susskind, B. Swingle, and Y. Zhao, Complexity, action, and black holes, Phys. Rev. D 93, 086006 (2016).

[74] W. J. Pan and Y. C. Huang, Holographic complexity and action growth in massive gravities, Phys. Rev. D 95, 126013 (2017).

[75] D. Momeni, S. A. H. Mansoori, and R. Myrzakulov, Holographic complexity in gauge/string superconductors, Phys. Lett. B 756, 354 (2016).

[76] S. Chapman, H. Marrochio, and R. C. Myers, Complexity of formation in holography, J. High Energy Phys. 01 (2017) 062.

[77] L. Lehner, R. C. Myers, E. Poisson, and R. D. Sorkin, Gravitational action with null boundaries, Phys. Rev. D 94, 084046 (2016).

[78] D. Carmi, R. C. Myers, and P. Rath, Comments on holographic complexity, J. High Energy Phys. 03 (2017) 118.

[79] J. Tao, P. Wang, and H. Yang, Testing holographic conjectures of complexity with Born-Infeld black holes, Eur. Phys. J. C 77, 817 (2017).
[80] M. Alishahiha, A. Faraji Astaneh, A. Naseh, and M. H. Vahidinia, On complexity for $\mathrm{F}(\mathrm{R})$ and critical gravity, J. High Energy Phys. 05 (2017) 009.

[81] A. Reynolds and S. F. Ross, Complexity in de Sitter space, Classical Quantum Gravity 34, 175013 (2017).

[82] M. M. Qaemmaqami, Complexity growth in minimal massive 3D gravity, Phys. Rev. D 97, 026006 (2018).

[83] W. D. Guo, S. W. Wei, Y. Y. Li, and Y. X. Liu, Complexity growth rates for AdS black holes in massive gravity and $f(R)$ gravity, Eur. Phys. J. C 77, 904 (2017).

[84] Y. G. Miao and L. Zhao, Complexity-action duality of the shock wave geometry in a massive gravity theory, Phys. Rev. D 97, 024035 (2018).

[85] L. Sebastiani, L. Vanzo, and S. Zerbini, Action growth for black holes in modified gravity, Phys. Rev. D 97, 044009 (2018).

[86] J. Couch, S. Eccles, W. Fischler, and M.-L. Xiao, Holographic complexity and noncommutative gauge theory, J. High Energy Phys. 03 (2018) 108.

[87] B. Swingle and Y. Wang, Holographic complexity of Einstein-Maxwell-dilaton gravity, J. High Energy Phys. 09 (2018) 106.

[88] P. A. Cano, R. A. Hennigar, and H. Marrochio, Complexity Growth Rate in Lovelock Gravity, Phys. Rev. Lett. 121, 121602 (2018).

[89] S. Chapman, H. Marrochio, and R. C. Myers, Holographic complexity in Vaidya spacetimes. Part I, J. High Energy Phys. 06 (2018) 046.

[90] S. Chapman, H. Marrochio, and R. C. Myers, Holographic complexity in Vaidya spacetimes. Part II, J. High Energy Phys. 06 (2018) 114.

[91] R. Fareghbal and P. Karimi, Complexity growth in flatland, Phys. Rev. D 98, 046003 (2018).

[92] R. Auzzi, S. Baiguera, M. Grassi, G. Nardelli, and N. Zenoni, Complexity and action for warped AdS black holes, J. High Energy Phys. 09 (2018) 013.

[93] H. Ghaffarnejad, E. Yaraie, and M. Farsam, Complexity growth and shock wave geometry in AdS-Maxwell-powerYang-Mills theory, Eur. Phys. J. C 78, 967 (2018).

[94] M. Alishahiha, A. Faraji Astaneh, M. R. Mohammadi Mozaffar, and A. Mollabashi, Complexity growth with Lifshitz scaling and hyperscaling violation, J. High Energy Phys. 07 (2018) 042.

[95] Y.S. An and R. H. Peng, Effect of the dilaton on holographic complexity growth, Phys. Rev. D 97, 066022 (2018).

[96] R. G. Cai, S. M. Ruan, S. J. Wang, R. Q. Yang, and R. H. Peng, Action growth for AdS black holes, J. High Energy Phys. 09 (2016) 161.

[97] C. Krishnan and A. Raju, A Neumann boundary term for gravity, Mod. Phys. Lett. A 32, 1750077 (2017).

[98] K. Parattu, S. Chakraborty, B. R. Majhi, and T. Padmanabhan, A boundary term for the gravitational action with null boundaries, Gen. Relativ. Gravit. 48, 94 (2016).

[99] A. Reynolds and S. F. Ross, Divergences in holographic complexity, Classical Quantum Gravity 34, 105004 (2017).

[100] S. Chakraborty, Boundary terms of the Einstein-Hilbert action, Fundam. Theor. Phys. 187, 43 (2017). 
[101] R. Q. Yang, C. Niu, and K. Y. Kim, Surface counterterms and regularized holographic complexity, J. High Energy Phys. 09 (2017) 042.

[102] W. C. Gan and F. W. Shu, Holographic complexity: A tool to probe the property of reduced fidelity susceptibility, Phys. Rev. D 96, 026008 (2017).

[103] S. Chakraborty, K. Parattu, and T. Padmanabhan, A novel derivation of the boundary term for the action in LanczosLovelock gravity, Gen. Relativ. Gravit. 49, 121 (2017).

[104] S. Chakraborty and K. Parattu, Null boundary terms for Lanczos-Lovelock gravity, arXiv:1806.08823.

[105] J. Jiang and H. Zhang, Surface term, corner term, and action growth in $\mathrm{F}($ Riemann) gravity theory, arXiv: 1806.10312.

[106] D. Momeni, M. Faizal, S. Bahamonde, and R. Myrzakulov, Holographic complexity for time-dependent backgrounds, Phys. Lett. B 762, 276 (2016).

[107] H. Huang, X. H. Feng, and H. Lu, Holographic complexity and two identities of action growth, Phys. Lett. B 769, 357 (2017).

[108] D. Carmi, S. Chapman, H. Marrochio, R. C. Myers, and S. Sugishita, On the time dependence of holographic complexity, J. High Energy Phys. 11 (2017) 188.

[109] R. Q. Yang, C. Niu, C. Y. Zhang, and K. Y. Kim, Comparison of holographic and field theoretic complexities for time dependent thermofield double states, J. High Energy Phys. 02 (2018) 082.

[110] M. Ghodrati, Complexity growth in massive gravity theories, the effects of chirality, and more, Phys. Rev. D 96, 106020 (2017).

[111] J. Jiang, Action growth rate for a higher curvature gravitational theory, Phys. Rev. D 98, 086018 (2018).

[112] A. R. Brown and L. Susskind, Second law of quantum complexity, Phys. Rev. D 97, 086015 (2018).

[113] S. Lloyd, Ultimate physical limits to computation, Nature (London) 406, 1047 (2000).

[114] M. Moosa, Evolution of complexity following a global quench, J. High Energy Phys. 03 (2018) 031.

[115] Z. Fu, A. Maloney, D. Marolf, H. Maxfield, and Z. Wang, Holographic complexity is nonlocal, J. High Energy Phys. 02 (2018) 072.

[116] F. J. G. Abad, M. Kulaxizi, and A. Parnachev, On complexity of holographic flavors, J. High Energy Phys. 01 (2018) 127.

[117] D. S. Ageev and I. Y. Arefeva, Holography and nonlocal operators for the BTZ black hole with nonzero angular momentum, Theor. Math. Phys. 180, 881 (2014).

[118] D. Ageev, I. Aref'eva, A. Bagrov, and M. I. Katsnelson, Holographic local quench and effective complexity, J. High Energy Phys. 08 (2018) 071.
[119] S. S. Gubser, Drag force in AdS/CFT, Phys. Rev. D 74, 126005 (2006).

[120] C. P. Herzog, A. Karch, P. Kovtun, C. Kozcaz, and L. G. Yaffe, Energy loss of a heavy quark moving through $N=4$ supersymmetric Yang-Mills plasma, J. High Energy Phys. 07 (2006) 013.

[121] J. Casalderrey-Solana and D. Teaney, Heavy quark diffusion in strongly coupled $\mathcal{N}=4$ Yang-Mills theory, Phys. Rev. D 74, 085012 (2006).

[122] H. Liu, K. Rajagopal, and U. A. Wiedemann, Calculating the Jet Quenching Parameter from AdS/CFT, Phys. Rev. Lett. 97, 182301 (2006).

[123] K. B. Fadafan, H. Liu, K. Rajagopal, and U. A. Wiedemann, Stirring strongly coupled plasma, Eur. Phys. J. C 61, 553 (2009).

[124] K. B. Fadafan and H. Soltanpanahi, Energy loss in a strongly coupled anisotropic plasma, J. High Energy Phys. 10 (2012) 085.

[125] M. Atashi, K. B. Fadafan, and M. Farahbodnia, Holographic energy loss in non-relativistic backgrounds, Eur. Phys. J. C 77, 175 (2017).

[126] K. Nagasaki, Complexity of $\mathrm{AdS}_{5}$ black holes with a rotating string, Phys. Rev. D 96, 126018 (2017).

[127] M. Banados, C. Teitelboim, and J. Zanelli, Black Hole in Three-Dimensional Spacetime, Phys. Rev. Lett. 69, 1849 (1992).

[128] A. Nata Atmaja and K. Schalm, Anisotropic drag force from 4D Kerr-AdS black holes, J. High Energy Phys. 04 (2011) 070.

[129] A. P. Reynolds and S. F. Ross, Butterflies with rotation and charge, Classical Quantum Gravity 33, 215008 (2016).

[130] A. M. Awad and C. V. Johnson, Higher dimensional Kerr-AdS black holes and the AdS/CFT correspondence, Phys. Rev. D 63, 124023 (2001).

[131] H. Lu, J. Mei, and C. N. Pope, Kerr/CFT correspondence in diverse dimensions, J. High Energy Phys. 04 (2009) 054.

[132] S. W. Hawking, C. J. Hunter, and M. M. Taylor-Robinson, Rotation and the AdS/CFT correspondence, Phys. Rev. D 59, 064005 (1999).

[133] S. W. Hawking and H. S. Reall, Charged and rotating AdS black holes and their CFT duals, Phys. Rev. D 61, 024014 (1999).

[134] Y. D. Tsai, X. N. Wu, and Y. Yang, Phase structure of KerrAdS black hole, Phys. Rev. D 85, 044005 (2012).

[135] A. Ovgun and K. Jusufi, Complexity growth rates for AdS black holes with dyonic/nonlinear charge/stringy hair/ topological defects, arXiv:1801.09615.

[136] R. G. Cai, M. Sasaki, and S. J. Wang, Action growth of charged black holes with a single horizon, Phys. Rev. D 95, 124002 (2017). 